astro-ph/9808196

DSF $30 / 98$

\title{
Precision Rates for Nucleon Weak Interactions in Primordial Nucleosynthesis and ${ }^{4} \mathrm{He}$ abundance
}

\author{
S. Esposito, G. Mangano, G. Miele and O. Pisanti, \\ Dipartimento di Scienze Fisiche, Universitá di Napoli "Federico II", and INFN, Sezione \\ di Napoli, Mostra D'Oltremare Pad. 20, I-80125 Napoli, Italy
}

\begin{abstract}
We report the results of a detailed calculation of nucleon weak interactions relevant for the neutron to proton density ratio at the onset of primordial nucleosynthesis. Radiative electromagnetic corrections, finite nucleon mass terms, thermal radiative effects on weak processes and on neutrino temperature are taken into account to reduce the theoretical uncertainty on $n \leftrightarrow p$ rates to $1 \%$. This translates into a sensitivity in ${ }^{4} \mathrm{He}$ mass fraction $Y_{p}$ prediction up to $10^{-4}$. We find a positive total correction to the Born prediction $\delta Y_{p} \simeq 0.004$.
\end{abstract}

PACS number(s): 98.80.Cq; 95.30.Cq; 11.10.Wx; 13.40.Ks 


\section{Introduction}

Primordial Big Bang Nucleosynthesis (BBN) [1], 2], represents one of the greatest success of the hot Big Bang Model and is among the main achievements of the interplay of particle physics and cosmology. The predicted abundances of all light nuclei produced in the early stage of the life of the universe, only depend on the baryon to photon density ratio $\eta$, and a single value for $\eta \sim 10^{-10}$ may accomodate all observed order of magnitude for ${ }^{4} \mathrm{He}, \mathrm{D}$, ${ }^{3} \mathrm{He}$ and ${ }^{7} \mathrm{Li}$ data. In the last few years, due to the increasing precision in observations [3], an even more exciting possibility can be foreseen, namely that BBN may tell us new pieces of information on both fundamental physics beyond the Standard Model and our present understanding of the evolution of the universe. In this respect it is already remarkable that $\mathrm{BBN}$ has been able to strongly constraint the number of effective neutrino degrees of freedom. This perspective, however, the fact that BBN is now entering in his maturity and precision era, demands similar improvements in the precision of the theoretical analysis, in order to reduce as much as possible all uncertainties on the predictions. An increasing precision in the measurements of ${ }^{4} \mathrm{He}$ mass fraction at the level of $10^{-4}$ [4] requires, for example, a reliability of proton-neutron conversion rates at the level of one percent at least, and a control at the same level of precision of all other effects which are relevant for the neutron to proton ratio at the freeze-out.

BBN took place at temperatures in the range $0.1 \div 10 \mathrm{MeV}$ and can be seen as the final outcome of two stages, the decoupling of the weak interactions which keep neutrons and protons in chemical equilibrium and, shortly after, the onset of nuclear reactions which start producing light nuclear species. At temperature $T>>\mathrm{MeV}$ the neutron to proton ratio is order unity and starts decreasing exponentially when the temperature reaches the value of their mass difference. As the temperature decreases, however, weak interactions are no longer fast enough to maintain equilibrium and a substantial final neutron fraction survives down to the phase of nucleosynthesis. All neutrons become practically bound in ${ }^{4} \mathrm{He}$ nuclei, due to the high binding energy per nucleon, which therefore represent the dominant products of $\mathrm{BBN}$, with an abundance in mass $Y_{p}$ close to $25 \%$. While $Y_{p}$ is weakly depending on the baryon to photon ratio $\eta$, it is instead strongly influenced by 
the ratio $n / p$ at the freeze-out, so it is particularly relevant to have a good theoretical estimate of this quantity.

The nuclear reaction chain starts with deuterium formation via $p+n \rightarrow D+\gamma$ when the inverse rate for Deuterium dissociation starts falling. This happens when the temperature has decreased below the tiny $\mathrm{D}$ binding energy $E_{B}=2.2 \mathrm{MeV}$ and, due to the large photon to baryon density ratio, when even the tail of photon Bose distribution becomes ineffective in D photodissociation, i.e. $\eta^{-1} \exp \left(-E_{B} / T\right) \sim 1$, which gives $T \sim 0.1 \mathrm{MeV}$. After Deuterium, the nuclear chain starts, leading to sensible production of nuclei up to ${ }^{7} \mathrm{Li}$ (for a recent review see [5]).

The more recent data on ${ }^{4} \mathrm{He}$ mass fraction and D abundance are still controversial since there are two different sets of results mutually incompatible [4]

$$
\begin{aligned}
& Y_{p}=0.234 \pm 0.0054, \quad D / H=(1.9 \pm 0.4) \cdot 10^{-4} ; \\
& Y_{p}=0.243 \pm 0.003, \quad D / H=(3.40 \pm 0.25) \cdot 10^{-5}
\end{aligned}
$$

which show the well known fact that large Helium mass fractions requires low values for Deuterium abundance and viceversa. It is possible that forthcoming measurements from high-redshift, low-metallicity QSO, or better understanding of the present data will clarify the situation [3], either in solving the controversy or pointing out a difficulty of standard $\mathrm{BBN}$ scenario. The fact which however is emerging from the above results is that Helium data are now reaching a precision of one per mille, requiring a similar effort in reducing the theoretical uncertainties on elementary $n \leftrightarrow p$ processes, as well as in evaluating the effect of uncertainties in the experimental nuclear reaction rates [6].

The now classical numerical codes which are used to deduce nuclei abundances [7], have been already modified by several authors, in order to include corrections to the tree level $n \leftrightarrow p$ transition rates. These corrections have distinct nature and, listed as they were historically introduced, are the following.

First of all, when accuracy of the order of one percent is required, all Born amplitudes for $n \leftrightarrow p$ processes should be corrected for order $\alpha$ radiative corrections. These effects have been extensively studied in literature [8]-11] and can be classified in outer factors, involving the nucleon as a whole, and inner ones, which instead depend on the details 
of nucleon internal structure. Their inclusion in the $\beta$-decay rate reduces the tree level estimate of neutron lifetime $\tau_{n} \simeq 961 \mathrm{sec}$ to the value $\tau_{n} \simeq 894 \mathrm{sec}$, which is closer to the experimental one $\tau_{n}^{e x p}=886.7 \pm 1.9 \mathrm{sec}$ [12]. A similar correction, of the order of $10 \%$, is expected to affect the rates of all elementary processes relevant for BBN

$$
\begin{array}{ccc}
\text { (a) } \nu_{e}+n \rightarrow e^{-}+p & , & \text { (d) } \bar{\nu}_{e}+p \rightarrow e^{+}+n \\
\text { (b) } e^{-}+p \rightarrow \nu_{e}+n & , & \text { (e) } n \rightarrow e^{-}+\bar{\nu}_{e}+p \\
\text { (c) } e^{+}+n \rightarrow \bar{\nu}_{e}+p & , & \text { (f) } e^{-}+\bar{\nu}_{e}+p \rightarrow n
\end{array}
$$

Other small effects are actually expected at higher order, since the estimated value of $\tau_{n}$ is compatible with the experimental value at 4- $\sigma$ level only. These additional contributions are usually taken into account by eliminating the coupling in front of the reaction rates $(a)-(f)$ in favour of $\tau_{n}^{e x p}$.

It was quite soon realized [13, 14] that since all reactions (1.2) take place in a thermal bath of electron, positron, neutrinos, antineutrinos and photons, finite density and temperature radiative effects should be included as well. They may affect the Born rates for factors of the order $\alpha T / m_{e}$, with $m_{e}$ the electron mass, which in the temperature interval relevant for BBN may be as large as few percent. Calculations of these effects, using the real time formalism of finite temperature field theory [15], has been done by several authors [13, 14], [16]-27]. Though they agree on the magnitude of the induced effect on ${ }^{4} \mathrm{He}$ abundance, there are however striking differences in the sign of the corrections.

Thermal effects play also a role in modifying neutrino temperature after decoupling. The difference between neutrino and photon temperatures originates from the entropy release to photon in $e^{+}-e^{-}$pair annihilation which, roughly speaking, takes place at temperature of the order of the electron mass $m_{e}$, shortly after neutrino decoupling. The interaction with the surrounding medium gives an extra plasma mass term to $e^{+}-e^{-}$, of the order of $\alpha T^{2} / m_{e}$, which slightly changes the neutrino to photon temperature ratio. Actually it has been shown that there is a residual small coupling of neutrinos at the electron-positron annihilation phase, which introduces non equilibrium effects in the neutrino energy distribution [13], 28]. This last effect introduces corrections to ${ }^{4} \mathrm{He}$ abundance of the order of $(1 \div 2) \cdot 10^{-4}$. 
Finally all Born amplitudes for the processes in (1.2) should be also corrected for nucleon finite mass effects. This has been first considered in [29]. They affect the allowed phase space as well as the weak amplitudes, which should now include the contribution of nucleon weak magnetism. Initial nucleons with finite mass will also have a thermal distribution in the comoving frame, over which it is necessary to average the transition probabilities.

In this paper we have considered in detail all the above effects, with the only exception of non equilibrium distortion of neutrino distribution, whose contribution to ${ }^{4} \mathrm{He}$ mass fraction is however very small. In particular:

i) all radiative corrections have been included at order $\alpha$;

ii) thermal radiative effects have been recalculated;

iii) all finite nucleon mass corrections at order $1 / M_{N}$ are considered.

The motivation for such an analysis which, as we have stressed, is certainly not new, is twofold. On one side for some of these effects different results have been obtained by several authors. Furthermore the precision expected for ${ }^{4} \mathrm{He}$ mass fraction measurements requires a careful scrutiny of the net effect of all these one percent level corrections.

We have organized the paper as follows. In section 2 we report the customary ex-

pressions for Born rates for the processes (1.2) . Section 3 is devoted to radiative zero temperature corrections. In section 4 we discuss finite nucleon mass corrections, while in section 5 we report all our results for thermal radiative effects, evaluated in the real time formalism. In section 6 we present our results and discuss the expected correction to ${ }^{4} \mathrm{He}$ mass fraction. Finally in section 7 we report our conclusions and outlook.

\section{The Born rates for $n \leftrightarrow p$ reactions}

At the epoch of BBN, $0.1 \mathrm{MeV} \leq T \leq 10 \mathrm{MeV}$, the universe was filled by a primordial plasma of nucleons, electrons, positrons, neutrinos, antineutrinos and photons, which were initially in thermal equilibrium. The initial conditions for the primordial nucleosynthesis are settled out by the ratio of the relative abundances of neutrons and protons. For 
temperature larger than $1 \mathrm{MeV}$ this ratio is strongly fixed by thermodynamical equilibrium, but as $T$ continues decreasing the necessary conditions to sustain the equilibrium are no longer satisfied. In this regime, to obtain the neutron-proton abundances, it is necessary to solve the Boltzmann equation, where the rates for the charged-current weak interactions transforming $n \leftrightarrow p([1.2)$ are involved. Shortly after, when the temperature drops down to $0.1 \div 0.3 \mathrm{MeV}$, the nuclear reactions become strongly active and transform neutrons mainly in ${ }^{4} \mathrm{He}$, and only in a small fraction in the other light elements.

To obtain the rates per nucleon for the $n \leftrightarrow p$ processes, let us consider a generic transition $i \rightarrow f$ in (1.2) . The differential reaction rate can be written in the form

$$
\begin{aligned}
d \Gamma(i \rightarrow f) & =\sum_{\text {spins }}|M(i \rightarrow f)|^{2} \prod_{i} \frac{d^{3} \mathbf{p}_{i}}{(2 \pi)^{3} 2 p_{0 i}} F_{i} \prod_{f} \frac{d^{3} \mathbf{p}_{f}}{(2 \pi)^{3} 2 p_{0 f}}\left(1-F_{f}\right) \\
& \times(2 \pi)^{4} \delta^{(4)}\left(\sum_{i} p_{i}-\sum_{f} p_{f}\right),
\end{aligned}
$$

where $|M(i \rightarrow f)|^{2}$ is the squared matrix element, to be summed over all spin degrees of freedom, the four-momentum of $j$-th particle is $p_{j}=\left(p_{0 j}, \mathbf{p}_{j}\right)$, and $F_{j}$ denotes the phase space density of the $j$-th particle.

Since the temperatures involved during the BBN are less than $10 \mathrm{MeV}$, in first approximation one can neglect in (2.1) the kinetic energy of nucleons with respect to their mass. Moreover the Pauli blocking factor for the final nucleon is completely negligible, since nucleons are strongly nonrelativistic and $F_{N_{f}} \leq \exp \left(-M_{N_{f}} / T\right) \leq \exp (-100)$, where $N_{f}$ denotes the final nucleon. In this limit, the differential reaction rate per incident nucleon $\left(N_{i}\right)$ becomes

$$
\begin{aligned}
d \omega_{B}(i \rightarrow f) & =2 \pi \sum_{\text {spins }} \frac{|M(i \rightarrow f)|^{2}}{8 M_{N_{i}} M_{N_{f}}} \prod_{i \neq N_{i}} \frac{d^{3} \mathbf{p}_{i}}{(2 \pi)^{3} 2 p_{0 i}} F_{i} \prod_{f \neq N_{f}} \frac{d^{3} \mathbf{p}_{f}}{(2 \pi)^{3} 2 p_{0 f}}\left(1-F_{f}\right) \\
& \times \delta\left(M_{N_{i}}-M_{N_{f}}+\sum_{i \neq N_{i}} p_{0 i}-\sum_{f \neq N_{f}} p_{0 f}\right),
\end{aligned}
$$

Hereafter, we will denote with $\omega(i \rightarrow f)$ the reaction rate per incident nucleon for the process $i \rightarrow f$ and the index $B$ reminds that the rates are computed at tree level and in the infinite mass limit for nucleons. We will refer to this as the Born approximation.

Let us start considering as an example the reaction (a) in (1.2) . At tree level, the 
corresponding $V-A$ amplitude for the diagram of Figure 1 is

$$
M\left(\nu_{e}+n \rightarrow e^{-}+p\right)=\frac{G_{F}}{\sqrt{2}} \bar{u}_{p}(p) \gamma_{\mu}\left(C_{V}-C_{A} \gamma_{5}\right) u_{n}\left(q^{\prime}\right) \bar{u}_{e}\left(p^{\prime}\right) \gamma^{\mu}\left(1-\gamma_{5}\right) u_{\nu}(q)
$$

where $G_{F}$ is the Fermi coupling constant and $C_{V}, C_{A}$ are the vector and axial coupling of the nucleon. In Born approximation we have for (2.3)

$$
\sum_{\text {spins }} \frac{|M|^{2}}{8 M_{n} M_{p}}=4 G_{F}^{2}\left[\left(C_{V}^{2}+3 C_{A}^{2}\right) p_{0}^{\prime} q_{0}+\left(C_{V}^{2}-C_{A}^{2}\right) \mathbf{p}^{\prime} \cdot \mathbf{q}\right]
$$

Thus, by substituting (2.4) in (2.2) and after integrating we get

$$
\omega_{B}\left(\nu_{e}+n \rightarrow e^{-}+p\right)=\frac{G_{F}^{2}\left(C_{V}^{2}+3 C_{A}^{2}\right)}{2 \pi^{3}} \int_{0}^{\infty} d\left|\mathbf{p}^{\prime}\right|\left|\mathbf{p}^{\prime}\right|^{2} q_{0}^{2} \Theta\left(q_{0}\right) F_{\nu}\left(q_{0}\right)\left[1-F_{e}\left(p_{0}^{\prime}\right)\right]
$$

where the integration limits are imposed by the $\Theta$-function, $q_{0} \geq 0$. In the present case we have $q_{0}=-\left(M_{n}-M_{p}\right)+p_{0}^{\prime} \equiv-\Delta+p_{0}^{\prime}$. The Fermi statistical distributions for electrons and neutrinos in the comoving frame are 円

$$
F_{e}\left(p_{0}^{\prime}\right)=\left[e^{\beta\left|p_{0}^{\prime}\right|}+1\right]^{-1}, \quad F_{\nu}\left(q_{0}\right)=\left[e^{\beta_{\nu}\left|q_{0}\right|}+1\right]^{-1},
$$

with $\beta=1 / T$ and $\beta_{\nu}=1 / T_{\nu}$.

Due to crossing symmetry, for the other five processes in (1.2) the form of $\sum_{\text {spins }}|M|^{2}$ in (2.2) remains unchanged, so the final result can be obtained from (2.5) by simply replacing $q_{0}$, determined by the energy conservation for each reaction, and the thermal factors, which depend on the initial and final lepton states for each process. In Table 1, using the same notation adopted for $\nu_{e}+n \rightarrow e^{-}+p$, we report the values for $q_{0}$ and the thermal factors for each reaction in (1.2), while in Figure 2 we show the Born rates for processes (1.2) versus photon temperature.

The electron neutrino temperature behaviour after decoupling is shortly reviewed in Appendix A. The result quoted there for the ratio $T_{\nu} / T$ will be used all through our analysis. Non-equilibrium effects on neutrino distributions due to residual coupling during the $e^{+}-$ $e^{-}$annihilation phase, and their implications on ${ }^{4} \mathrm{He}$ abundance will be shortly discussed in section 6 .

\footnotetext{
${ }^{1}$ We are assuming vanishing chemical potential for neutrinos. See [0] for a review on the effects induced by neutrino asymmetry and constraints on chemical potentials.
} 
Table 1.

\begin{tabular}{|c|r|c|}
\hline \hline Reactions & \multicolumn{1}{|c|}{$q_{0}$} & Thermal factors \\
\hline \hline$\nu_{e}+n \rightarrow e^{-}+p$ & $-\Delta+p_{0}^{\prime}$ & $F_{\nu}\left(q_{0}\right)\left(1-F_{e}\left(p_{0}^{\prime}\right)\right)$ \\
$e^{-}+p \rightarrow \nu_{e}+n$ & $-\Delta+p_{0}^{\prime}$ & $F_{e}\left(p_{0}^{\prime}\right)\left(1-F_{\nu}\left(q_{0}\right)\right)$ \\
$e^{+}+n \rightarrow \bar{\nu}_{e}+p$ & $\Delta+p_{0}^{\prime}$ & $F_{e}\left(p_{0}^{\prime}\right)\left(1-F_{\nu}\left(q_{0}\right)\right)$ \\
$\bar{\nu}_{e}+p \rightarrow e^{+}+n$ & $\Delta+p_{0}^{\prime}$ & $F_{\nu}\left(q_{0}\right)\left(1-F_{e}\left(p_{0}^{\prime}\right)\right)$ \\
$n \rightarrow e^{-}+\bar{\nu}_{e}+p$ & $\Delta-p_{0}^{\prime}$ & $\left(1-F_{\nu}\left(q_{0}\right)\right)\left(1-F_{e}\left(p_{0}^{\prime}\right)\right)$ \\
$e^{-}+\bar{\nu}_{e}+p \rightarrow n$ & $\Delta-p_{0}^{\prime}$ & $F_{\nu}\left(q_{0}\right) F_{e}\left(p_{0}^{\prime}\right)$ \\
\hline \hline
\end{tabular}

\section{Radiative electromagnetic corrections: the neutron lifetime}

In order to measure the level of validity of Born approximation in the evaluation of rates (1.2), one can use the corresponding prediction for neutron lifetime. By using (2.5) and Table 1, in the limit of vanishing density one gets the simple expression

$$
\tau_{n}^{-1}=\frac{G_{F}^{2}\left(C_{V}^{2}+3 C_{A}^{2}\right)}{2 \pi^{3}} m_{e}^{5} \int_{1}^{\frac{\Delta}{m_{e}}} d \epsilon \epsilon\left(\epsilon-\frac{\Delta}{m_{e}}\right)^{2}\left(\epsilon^{2}-1\right)^{\frac{1}{2}} .
$$

Thus, inserting the value of $G_{F}$, very well known from the measurement of muon decay [12], $C_{V}=V_{u d}=0.9751 \pm 0.0006$ and $C_{A} / C_{V}=1.2601 \pm 0.0025$, deduced from the study of the decay product angular distribution in neutron decay [12], we obtain the value $\tau_{n} \simeq 961 \mathrm{sec}$ which has to be compared with the experimental result $\tau_{n}^{e x p}=886.7 \pm 1.9 \mathrm{sec}$ [12]. As a consequence, a correction factor to (3.1) of about $8 \%$ is needed. Note that similar corrections are expected to affect the other reactions in (1.2) as well, since they are mediated by the same interaction hamiltonian.

Let us consider order $\alpha$ QED corrections to neutron lifetime ( $\alpha$ is the fine structure constant); these can be separated into outer corrections, involving the nucleon as a whole, and inner corrections, depending on nucleon structure. Obviously, the inner corrections are sensible to the details of the strong interactions inside the nucleon, while it can be shown 8 that outer corrections, at least up to terms of order $\alpha$, take a general form. 
In terms of Feynman diagrams the corrections at order $\alpha$ to neutron lifetime are obtained from the pure $V-A$ interaction (2.3) with the exchange of a photon between the four fermions involved. Note that nucleons interact electromagnetically through both their charge and magnetic moment, even though the interactions involving magnetic moments are suppressed by the inverse of the nucleon mass.

The outer correction to the differential decay rate can be written as the multiplicative factor

$$
\left[1+\frac{\alpha}{2 \pi} g\left(p_{0}^{\prime}, q_{0}\right)\right]
$$

where $g\left(p_{0}^{\prime}, q_{0}\right)$ is a function of electron and neutrino energy, whose explicit expression can be found in eq. (20b) of Ref. [8]. This function describes, at order $\alpha$, the deviations in the allowed electron spectrum arising from the radiative corrections? It increases the decay probability for neutron $\beta$-decay (and therefore decreases neutron lifetime) by about $1.6 \%$.

Inner corrections are much more difficult to handle since they strictly depend on nucleon structure. In general, one can follow two different approaches. One possibility is to directly consider radiative corrections to the effective nucleon weak current $\bar{u}_{p} \gamma_{\mu}(1-$ $\left.\left(C_{A} / C_{V}\right) \gamma_{5}\right) u_{n}$ [30]. Alternatively, one can study corrections to quark currents $\bar{q} \gamma_{\mu}\left(1-\gamma_{5}\right) q$ and then translate the quark-based description into the hadronic one [9]. Here, we adopt the second point of view, and report the results obtained by Marciano and Sirlin [9]

$$
\frac{\alpha}{2 \pi}\left(4 \ln \frac{M_{Z}}{M_{p}}+\ln \frac{M_{p}}{M_{A}}+2 C+A_{g}\right) .
$$

The first term represents the dominant model-independent short-distance contribution. The second and third terms are axial-current induced contributions, where $M_{A}$ is a low energy cutoff applied to the short-distance part of the $\gamma W$ box diagram and $2 C$ is the remaining long-distance (low energy) correction. These terms depend on the details of the strong interaction structure and are the main sources of uncertainty on the radiative corrections. The allowed range for the cutoff $M_{A}$ is $400 \div 1600 \mathrm{MeV}$, while $C=0.798 C_{A}\left(1+\mu_{p}+\mu_{n}\right)$,

\footnotetext{
${ }^{2}$ It is customary to substitute the function $g\left(p_{0}^{\prime}, q_{0}\right)$ in 3.2$)$ with its mean value $\bar{g}$ obtained averaging over the allowed electron spectrum. However, since we will consider not only neutron decay, but also the other five reactions in (1.2), we will not use this approximation.
} 
$\mu_{p}+\mu_{n} \simeq-0.12$ being the nucleon isoscalar anomalous magnetic moment. Then we have

$$
\frac{\alpha}{2 \pi}\left(\ln \frac{M_{p}}{M_{A}}+2 C\right) \simeq 0.0012 \pm 0.0018
$$

The last term in $(3.3)$ is a perturbative QCD correction whose calculation is rather reliable and gives $A_{g} \simeq-0.34$.

The largest correction comes from the first term in (3.3) and it seems appropriate to approximate the effects of higher orders by summing all leading-logarithmic corrections of the type $\alpha^{n} \ln ^{n}\left(M_{Z}\right)$ via a renormalization group analysis. This has been done in [10]; the corrected differential rate then acquires the multiplicative factor

$$
\mathcal{G}\left(p_{0}^{\prime}, q_{0}\right)=\left[1+\frac{\alpha}{2 \pi}\left(\ln \frac{M_{p}}{M_{A}}+2 C\right)+\frac{\alpha\left(M_{p}\right)}{2 \pi}\left[g\left(p_{0}^{\prime}, q_{0}\right)+A_{g}\right]\right] S\left(M_{p}, M_{Z}\right)
$$

where $\alpha(\mu)$ is the QED running coupling constant defined in the $\overline{M S}$ scheme. The shortdistance enhancement factor $S\left(M_{p}, M_{Z}\right)$ is given by [10, [9]

$$
S\left(M_{p}, M_{Z}\right)=\left(\frac{\alpha\left(m_{c}\right)}{\alpha\left(M_{p}\right)}\right)^{\frac{3}{4}}\left(\frac{\alpha\left(m_{\tau}\right)}{\alpha\left(m_{c}\right)}\right)^{\frac{9}{16}}\left(\frac{\alpha\left(m_{b}\right)}{\alpha\left(m_{\tau}\right)}\right)^{\frac{9}{19}}\left(\frac{\alpha\left(M_{W}\right)}{\alpha\left(m_{b}\right)}\right)^{\frac{9}{20}}\left(\frac{\alpha\left(M_{Z}\right)}{\alpha\left(M_{W}\right)}\right)^{\frac{36}{17}} .
$$

In the $\overline{M S}$ scheme the running coupling constant at different scales $\left(\alpha^{-1}\left(M_{Z}\right), \alpha^{-1}\left(M_{W}\right)\right.$, $\left.\alpha^{-1}\left(m_{b}\right), \alpha^{-1}\left(m_{\tau}\right), \alpha^{-1}\left(m_{c}\right), \alpha^{-1}\left(M_{p}\right)\right)$ is $(127.90,127.94,132.08,133.27,133.62,133.90)$ leading to $S\left(M_{p}, M_{Z}\right) \simeq 1.0225$.

The radiative corrections just considered, which are described by the factor in (3.5), are not, however, the only ones to be taken into account for the neutron decay. They contribute for about $4 \%$ to the lifetime, against the required global correction of about 8\%. The remaining leading corrections are usually viewed as corrections to the phase space factor [11], namely to the differential rate in (3.1), since they are electron and neutrino energy dependent.

Let us first examine the correction due to the distortion of the outgoing electron wave function by the Coulomb field of the proton. This can be calculated by solving the Dirac equation for an electron under the influence of a spatially finite proton charge distribution of radius $R \simeq 1 \mathrm{fm}$; the wave function is then evaluated at the centre of the proton. The correction factor is then given by [11]

$$
\mathcal{F}\left(p_{0}^{\prime}\right) \mathcal{L}\left(p_{0}^{\prime}\right) \simeq\left(1+\alpha \pi \frac{p_{0}^{\prime}}{\left|\mathbf{p}^{\prime}\right|}\right)\left[1-\alpha R p_{0}^{\prime}\left(1+\frac{m_{e}^{2}}{2{p_{0}^{\prime}}^{2}}\right)\right]
$$

\footnotetext{
${ }^{3}$ The expression reported in [9] has been updated to account for the measured top quark mass.
} 
The first term is usually denoted as the Fermi function for the Coulomb scattering.

Another small correction comes from the observation that neither the electron wave function, evaluated at the centre of the proton through $\mathcal{F}\left(p_{0}^{\prime}\right) \mathcal{L}\left(p_{0}^{\prime}\right)$, nor the anti-neutrino one are constant through the proton volume. Thus, the decay rate has to be computed by an appropriate convolution of the electron, anti-neutrino and proton wave functions through the proton volume. This leads to the following correction factor [11

$$
\mathcal{C}\left(p_{0}^{\prime}, q_{0}\right) \simeq 1+c_{0}+\frac{c_{1}}{p_{0}^{\prime}}+c_{2} p_{0}^{\prime}+c_{3} p_{0}^{2}
$$

with

$$
\begin{aligned}
c_{0} & =\frac{1}{5}\left[R^{2} m_{e}^{2}\left(1-\frac{2}{3} B-\frac{\left(p_{0}^{\prime}+q_{0}\right)^{2}}{m_{e}^{2}}\right)-\alpha B R\left(p_{0}^{\prime}+q_{0}\right)\right] \\
c_{1} & =\frac{2 B}{15} R^{2} m_{e}^{2}\left(p_{0}^{\prime}+q_{0}\right) \\
c_{2} & =\frac{R}{5}(3-B)\left[\frac{2}{3} R\left(p_{0}^{\prime}+q_{0}\right)-\alpha\right] \\
c_{3} & =\frac{2}{15}(3-B) R^{2} \\
B & =\frac{C_{V}^{2}-C_{A}^{2}}{\left(C_{V}^{2}+3 C_{A}^{2}\right)}
\end{aligned}
$$

Finally, there are also tiny corrections related to the fact that the outgoing proton is not at rest. An exhaustive discussion of finite nucleon mass corrections will be given in the following. Here, we only point out that since proton recoils, its Coulomb field is the one of a moving source. The correction associated to this effect is contained in the factor [1]

$$
\mathcal{Q}\left(p_{0}^{\prime}, q_{0}\right) \simeq 1-\frac{\alpha \pi m_{e}^{2}}{M_{p}\left|\mathbf{p}^{\prime}\right|}\left(1+B \frac{q_{0}}{3 p_{0}^{\prime}}\right) \quad
$$

We eventually get for the neutron $\beta$-decay differential rate, corrected at order $\alpha$

$$
d \omega\left(n \rightarrow e^{-}+\bar{\nu}_{e}+p\right)=\mathcal{G}\left(p_{0}^{\prime}, q_{0}\right) \mathcal{F}\left(p_{0}^{\prime}\right) \mathcal{L}\left(p_{0}^{\prime}\right) \mathcal{C}\left(p_{0}^{\prime}, q_{0}\right) \mathcal{Q}\left(p_{0}^{\prime}, q_{0}\right) d \omega_{B}\left(n \rightarrow e^{-}+\bar{\nu}_{e}+p\right)
$$

and thus the neutron lifetime reads

$$
\begin{aligned}
\tau_{n}^{-1} & =\frac{G_{F}^{2}\left(C_{V}^{2}+3 C_{A}^{2}\right)}{2 \pi^{3}} \int_{m_{e}}^{\Delta} d p_{0}^{\prime} p_{0}^{\prime}\left|\mathbf{p}^{\prime}\right|\left(p_{0}^{\prime}-\Delta\right)^{2} \\
& \times \mathcal{G}\left(p_{0}^{\prime}, q_{0}\right) \mathcal{F}\left(p_{0}^{\prime}\right) \mathcal{L}\left(p_{0}^{\prime}\right) \mathcal{C}\left(p_{0}^{\prime}, q_{0}\right) \mathcal{Q}\left(p_{0}^{\prime}, q_{0}\right)
\end{aligned}
$$

Concerning the relevance of the different corrections just considered, a comment is in turn. For seek of completeness we have reported an exhaustive description of all 
corrections at order $\alpha$, but as clearly appears from the explicit expressions of (3.5)-(3.14), the main contributions come from (3.5) and $\mathcal{F}\left(p_{0}^{\prime}\right)$ term in (3.7). All other contributions, contained in $\mathcal{L}\left(p_{0}^{\prime}\right), \mathcal{C}\left(p_{0}^{\prime}, q_{0}\right)$ and $\mathcal{Q}\left(p_{0}^{\prime}, q_{0}\right)$, are in fact much smaller and thus they can be safely neglected, since they are suppressed by a factors of the order $\Delta / \Lambda_{Q C D}$. Actually the $\alpha^{2}$ contributions coming from radiative and Coulomb effects are even larger, or of the same order of magnitude, than these terms, so they should be included for consistency if $\mathcal{L}\left(p_{0}^{\prime}\right), \mathcal{C}\left(p_{0}^{\prime}, q_{0}\right)$ and $\mathcal{Q}\left(p_{0}^{\prime}, q_{0}\right)$ are taken into account [11]. For the level of accuracy of our analysis it will be sufficient to include $\mathcal{F}\left(p_{0}^{\prime}\right)$ and $\mathcal{G}\left(p_{0}^{\prime}, q_{0}\right)$ at order $\alpha$ only.

Evaluating numerically the integral over the phase space we then obtain for the neutron lifetime the value $\tau_{n}^{t h}=893.9 \mathrm{sec}$, which is now compatible in about $4-\sigma$ with the experimental value. The theoretical prediction for neutron lifetime can be further refined by considering, for example, higher order terms in $\alpha$, like subleading corrections, or other small effects, such as residual average proton polarization due to parity non-conservation and so on. Some of these effects are briefly discussed in [11], but for our purposes we do not consider them, since their small contributions would be mainly energy independent and then account only for an overall factor. In fact, assuming that such residual improving would eventually yield a theoretical prediction for neutron lifetime fully compatible with the experimental value, it is meaningful, as far as the aim of the present investigation is concerned, to rescale the differential rate of neutron $\beta$-decay in (3.16) for the residual constant small factor $\tau_{n}^{\text {th }} / \tau_{n}^{\text {exp }}$. This is a standard procedure, which allows to overcome the problem of a precise determination of the coupling constant for weak interactions involved in BBN, expressing this overall factor in terms of the experimental value of neutron lifetime. We stress, however, that it is worth rescaling the rate only after all known corrections have been included, since this increases the accuracy of the prediction. In this way the ansatz that the residual correction be an overall factor and not, as it is reasonable to expect, a function of leptons energies, may introduce errors less than $1 \%$.

The analysis of radiative corrections for $\beta$-decay shows that their contribution to the rates for the processes in (1.2), relevant for $\mathrm{BBN}$, is expected to be as large as few percent. To reach accuracy of the order of one percent in rate evaluations it is

\footnotetext{
${ }^{4}$ For completeness we have also included corrections coming from the finite nucleon mass, which are considered in section 4 . However, these corrections are very small.
} 
therefore necessary to correct Born rates for both radiative and Coulomb effects. This last contribution, however, is only present when both electron and proton are present in the initial or final state, since it can be viewed as the electromagnetic rescattering of the two charged particles. This results can be also obtained diagrammatically, using the traditional separation of electromagnetic corrections into order $\alpha$ (radiative) and order $Z \alpha$ (Coulomb) contributions, though this separation for the elementary reactions in (1.2) is somehow arbitrary, since $Z=1$. In this way it is straightforward to show that no Coulomb corrections $\mathcal{F}\left(p_{0}^{\prime}\right)$ are present for the channels $e^{+}+n \leftrightarrow \bar{\nu}_{e}+p$ (see [13] and references therein).

Summarizing, zero temperature radiative corrections modify the Born elementary rates in the following way:

i) the radiative energy dependent factor $\mathcal{G}\left(p_{0}^{\prime}, q_{0}\right)$ reported in (3.5) is included in the differential rates for all processes $(a)-(f)$;

ii) the Coulomb factor $\mathcal{F}\left(p_{0}^{\prime}\right)(3.7)$ is introduced for processes $(a),(b),(e)$ and $(f)$;

iii) all rates are rescaled by the factor

$$
1+\delta_{\tau} \equiv \tau_{n}^{t h} / \tau_{n}^{e x p} \quad, \quad \delta_{\tau}=0.008
$$

representing an energy independent constant correction which, as we have discussed, should be included to reproduce the experimental value for neutron lifetime at zero density.

\section{Finite nucleon mass corrections}

In this section we consider the corrections to the Born rates for the $n \leftrightarrow p$ reactions (1.2) induced by relaxing the assumption of infinite mass for nucleons. This is a necessary improvement since the finite nucleon mass corrections, proportional to $m_{e} / M_{N}$ or $T / M_{N} \sim$ $\alpha$, are of the same order of magnitude of the radiative ones.

The main effect 29] of considering finite values for the nucleon masses are to allow for new effective extra couplings, such as weak magnetism, and to change both the evaluation of the spin summed squared matrix element and the allowed phase space. Eq. (2.4) is in 
fact only valid for nucleons at rest. Furthermore, the fact that all rates should be averaged over the Maxwell-Boltzmann distribution for the initial nucleon, induces corrections of order $T / M_{N}$. This kinematical correction will be considered separately.

\subsection{Corrections to the transition amplitude and phase space}

For definiteness let us consider the process shown in Figure 1. At first order in $1 / M_{N}$, the transition amplitude can be written as

$$
M=\frac{G_{F}}{\sqrt{2}} \bar{u}_{p}(p) O_{\mu} u_{n}\left(q^{\prime}\right) \bar{u}_{e}\left(p^{\prime}\right) \gamma^{\mu}\left(1-\gamma_{5}\right) u_{\nu}(q)
$$

where the effective nucleon-nucleon weak coupling is given in general by [31

$$
O_{\mu}=\gamma_{\mu}\left(C_{V}-C_{A} \gamma_{5}\right)+i \frac{f_{2}}{M_{N}} \sigma_{\mu \nu}\left(p-q^{\prime}\right)^{\nu}
$$

The form factor $f_{2}$ is the anomalous weak charged-current magnetic moment, and we have

neglected both scalar coupling, related to CVC breaking, and pseudoscalar coupling of the nucleon, whose small value is fixed by low-energy PCAC theorems [12], [31]. For the weak magnetism term $f_{2}$, we have from CVC, $f_{2}=V_{u d}\left(\mu_{p}-\mu_{n}\right) / 2=1.81 V_{u d}$. In general, the form-factors $C_{V}, C_{A}$ and $f_{2}$, are all dependent on the transferred four-momentum. However, at the energy scales relevant for the considered processes, this dependence can be safely neglected.

In appendix B we have reported the spin-summed squared modulus of the transition amplitude (4.1). The result is cast in a completely general form in order to allow for a straightforward generalization to all processes of (1.2). Specializing this result for the process $\nu_{e}+n \rightarrow e^{-}+p$, and including pure radiative effects analyzed in the previous section we get the following expression for the rate

$$
\begin{aligned}
\omega_{R}\left(\nu_{e}+n \rightarrow e^{-}+p\right) & =\frac{1+\delta_{\tau}}{128 \pi^{3} M_{n}} \int_{0}^{\infty} d q_{0} \int_{E_{\text {inf }}}^{E_{\text {sup }}} d p_{0}^{\prime} \sum_{\text {spins }}|M|^{2}\left(p_{0}^{\prime}, q_{0}\right) \\
& \times \mathcal{G}\left(p_{0}^{\prime}, q_{0}\right) \mathcal{F}\left(p_{0}^{\prime}\right) F_{\nu}\left(q_{0}\right)\left[1-F_{e}\left(p_{0}^{\prime}\right)\right] .
\end{aligned}
$$

where

$$
E_{i n f}=\frac{\left[\left(M_{n}+q_{0}\right)\left(M_{n}^{2}-M_{p}^{2}+m_{e}^{2}+2 q_{0} M_{n}\right)-2 q_{0} \xi\right]}{2 M_{n}\left(M_{n}+2 q_{0}\right)}
$$




$$
\begin{aligned}
E_{\text {sup }} & =\frac{\left[\left(M_{n}+q_{0}\right)\left(M_{n}^{2}-M_{p}^{2}+m_{e}^{2}+2 q_{0} M_{n}\right)+2 q_{0} \xi\right]}{2 M_{n}\left(M_{n}+2 q_{0}\right)}, \\
\xi & =\frac{1}{2} \sqrt{\left[M_{n}^{2}-M_{p}^{2}-m_{e}^{2}+2 q_{0} M_{n}\right]^{2}-4 m_{e}^{2} M_{p}^{2}}
\end{aligned}
$$

Notice that the squared amplitude is evaluated in the neutron rest frame, and that the result is not averaged over the neutron velocity distribution. This effect will be considered in the following section. In Figure 3 we plot the zero-temperature radiative correction $\Delta \omega_{R} \equiv \omega_{R}-\omega_{B}$ normalized to $(\alpha / \pi) \omega_{B}$ for the processes (1.2). Weak magnetism and finite nucleon mass effect on phase space are included. The constant shift correction $\pi \delta_{\tau} / \alpha$ (see eq. (3.17)) has been subtracted in order to show the pure radiative and Coulomb effects and finite mass corrections.

\subsection{Kinematical corrections}

Apart from mass corrections to the squared matrix element and allowed phase space, there are indeed additional contributions to the reaction rates which are of a purely kinematical nature, namely the ones connected to the kinetic energy carried by the initial nucleon in the comoving frame.

Let us consider the reaction $\nu_{e}+n \rightarrow e^{-}+p$ and, with the same notation of the previous sections, its rate per initial nucleon

$$
\begin{aligned}
& \omega\left(\nu_{e}+n \rightarrow e^{-}+p\right)=\frac{1}{n_{n}} \int \frac{d^{3} \mathbf{q}^{\prime}}{(2 \pi)^{3} 2 q_{0}^{\prime}} F_{n}\left(q_{0}^{\prime}\right)\left\{\int \frac{d^{3} \mathbf{p}}{(2 \pi)^{3} 2 p_{0}} \frac{d^{3} \mathbf{q}}{(2 \pi)^{3} 2 q_{0}} \frac{d^{3} \mathbf{p}^{\prime}}{(2 \pi)^{3} 2 p_{0}^{\prime}}\right. \\
&\left.\times(2 \pi)^{4} \delta^{(4)}\left(q+q^{\prime}-p-p^{\prime}\right) \sum_{\text {spins }}|M|^{2}\right\} F_{\nu}\left(q_{0}\right)\left[1-F_{e}\left(p_{0}^{\prime}\right)\right],
\end{aligned}
$$

where, as already mentioned, one can safely approximate $1-F_{p}\left(p_{0}\right) \simeq 1$ and

$$
n_{n}=2 \int \frac{d^{3} \mathbf{q}^{\prime}}{(2 \pi)^{3}} F_{n}\left(q_{0}^{\prime}\right)
$$

denotes the incident neutron number density. All quantities in (4.7) are evaluated in the comoving frame, where the plasma is at rest, which coincides with the initial nucleon rest frame only in the infinite nucleon mass limit. Let us first note that the quantity in brace brackets is a Lorentz invariant so that it can be equally evaluated in the neutron rest frame. If we therefore denote with $\tilde{q}_{0}$ and $\tilde{p}_{0}^{\prime}$ neutrino and electron energies in the 
neutron rest frame and consider the change of variables in the integral (4.7) $\left(q_{0}, p_{0}^{\prime}\right) \rightarrow$ $\left(\tilde{q}_{0}, \tilde{p}_{0}^{\prime}\right)$, the transformed expression in brace brackets is simply obtained by substituting $q_{0} \rightarrow \tilde{q}_{0}, p_{0}^{\prime} \rightarrow \tilde{p}_{0}^{\prime}$. To evaluate how electron and neutrino thermal distribution factor $F_{\nu}\left(q_{0}\right)\left[1-F_{e}\left(p_{0}^{\prime}\right)\right]$ transforms under this change of variable, a simple computation gives, up to $1 / M_{n}^{2}$ terms

$$
\begin{aligned}
& p_{0}^{\prime} \simeq \tilde{p}_{0}^{\prime}+\frac{\mathbf{q}^{\prime} \cdot \tilde{\mathbf{p}}^{\prime}}{M_{n}}+\frac{\left|\mathbf{q}^{\prime}\right|^{2}}{2 M_{n}^{2}} \tilde{p}_{0}^{\prime} \\
& q_{0} \simeq \tilde{q}_{0}+\frac{\mathbf{q}^{\prime} \cdot \tilde{\mathbf{q}}}{M_{n}}+\frac{\left|\mathbf{q}^{\prime}\right|^{2}}{2 M_{n}^{2}} \tilde{q}_{0}
\end{aligned}
$$

and thus one gets

$$
F_{\nu}\left(q_{0}\right)\left[1-F_{e}\left(p_{0}^{\prime}\right)\right]=F_{\nu}\left(\tilde{q}_{0}\right)\left[1-F_{e}\left(\tilde{p}_{0}^{\prime}\right)\right]+\delta\left[F_{\nu}\left(1-F_{e}\right)\right],
$$

where

$$
\begin{aligned}
& \delta\left[F_{\nu}\left(1-F_{e}\right)\right] \simeq F_{\nu}\left(\tilde{q}_{0}\right)\left[1-F_{e}\left(\tilde{p}_{0}^{\prime}\right)\right]\left\{\frac{\mathbf{q}^{\prime} \cdot \tilde{\mathbf{p}}^{\prime}}{M_{n} T} F_{e}\left(\tilde{p}_{0}^{\prime}\right)-\frac{\mathbf{q}^{\prime} \cdot \tilde{\mathbf{q}}}{M_{n} T_{\nu}}\left[1-F_{\nu}\left(\tilde{q}_{0}\right)\right]\right. \\
& +\frac{\left|\mathbf{q}^{\prime}\right|^{2}}{2 M_{n}^{2} T} \tilde{p}_{0}^{\prime} F_{e}\left(\tilde{p}_{0}^{\prime}\right)-\frac{\left|\mathbf{q}^{\prime}\right|^{2}}{2 M_{n}^{2} T_{\nu}} \tilde{q}_{0}\left[1-F_{\nu}\left(\tilde{q}_{0}\right)\right]-\frac{1}{2}\left(\frac{\mathbf{q}^{\prime} \cdot \tilde{\mathbf{p}}^{\prime}}{M_{n} T}\right)^{2} F_{e}\left(\tilde{p}_{0}^{\prime}\right)\left[1-2 F_{e}\left(\tilde{p}_{0}^{\prime}\right)\right] \\
& \left.\quad-\frac{\mathbf{q}^{\prime} \cdot \tilde{\mathbf{p}}^{\prime} \mathbf{q}^{\prime} \cdot \tilde{\mathbf{q}}}{M_{n}^{2} T T_{\nu}} F_{e}\left(\tilde{p}_{0}^{\prime}\right)\left[1-F_{\nu}\left(\tilde{q}_{0}\right)\right]+\frac{1}{2}\left(\frac{\mathbf{q}^{\prime} \cdot \tilde{\mathbf{q}}}{M_{n} T_{\nu}}\right)^{2}\left[1-F_{\nu}\left(\tilde{q}_{0}\right)\right]\left[1-2 F_{\nu}\left(\tilde{q}_{0}\right)\right]\right\} .
\end{aligned}
$$

By substituting (4.11) in (4.7) it is easy to see that the first term on the r.h.s. of (4.11) reproduces the results of the previous section. Hence, the new contribution, proportional to $\delta\left[F_{\nu}\left(1-F_{e}\right)\right]$ of $(4.12)$ is an additional $1 / M_{n}$ correction to the Born rate which vanishes for $\left|\mathbf{q}^{\prime}\right| / M_{n} \rightarrow 0$ and is of a purely kinematical origin. It is due to the fact that the comoving and the neutron rest frames are not coinciding, unless neutron are taken infinitely massive, and initial neutrons have a velocity distribution in the comoving frame, over which the reaction differential rates should be averaged.

Using that in the nonrelativistic limit

$$
F_{n}\left(q_{0}^{\prime}\right)=\left[\exp \left(\frac{q_{0}^{\prime}}{T}\right)+1\right]^{-1} \simeq \exp \left(-\frac{q_{0}^{\prime}}{T}\right) \simeq \exp \left(-\frac{M_{n}}{T}\right) \exp \left(-\frac{\left|\mathbf{q}^{\prime}\right|^{2}}{2 M_{n} T}\right)
$$

the kinematical correction is

$$
\begin{aligned}
& \Delta \omega_{K}\left(\nu_{e}+n \rightarrow e^{-}+\right.p)=\frac{e^{-\frac{M_{n}}{T}}}{n_{n}} \int \frac{d^{3} \mathbf{q}^{\prime}}{(2 \pi)^{3} 2 q_{0}^{\prime}} e^{-\frac{\left|\mathbf{q}^{\prime}\right|^{2}}{\left.2\right|_{n} T}}\left\{\int \frac{d^{3} \tilde{\mathbf{p}}}{(2 \pi)^{3} 2 \tilde{p}_{0}} \frac{d^{3} \tilde{\mathbf{q}}}{(2 \pi)^{3} 2 \tilde{q}_{0}} \frac{d^{3} \tilde{\mathbf{p}}^{\prime}}{(2 \pi)^{3} 2 \tilde{p}_{0}^{\prime}}\right. \\
&\left.\times(2 \pi)^{4} \delta^{(4)}\left(\tilde{q}+\tilde{q}^{\prime}-\tilde{p}-\tilde{p}^{\prime}\right) \sum_{\text {spins }}|M|^{2}\right\} \delta\left[F_{\nu}\left(1-F_{e}\right)\right] .
\end{aligned}
$$


Since $\delta\left[F_{\nu}\left(1-F_{e}\right)\right]$ is already a $1 / M_{n}$ correction, one can compute the above integral assuming for the quantity in brace brackets the infinite nucleon mass limit. According to this approximation, the dependence of the integrand function on $\mathbf{q}^{\prime}$ comes through the expression (4.12) of $\delta\left[F_{\nu}\left(1-F_{e}\right)\right]$ only.

Performing in (4.14) first the integral in $d^{3} \mathbf{q}^{\prime}$ and observing that

$$
\int \frac{d^{3} \mathbf{q}^{\prime}}{(2 \pi)^{3} 2 M_{n}} \exp \left\{-\frac{\left|\mathbf{q}^{\prime}\right|^{2}}{2 M_{n} T}\right\} \mathbf{q}^{\prime}=0
$$

and

$$
\frac{e^{-\frac{M_{n}}{T}}}{n_{n}} \int \frac{d^{3} \mathbf{q}^{\prime}}{(2 \pi)^{3} 2 M_{n}} \exp \left\{-\frac{\left|\mathbf{q}^{\prime}\right|^{2}}{2 M_{n} T}\right\} q_{i}^{\prime} q_{j}^{\prime}=\frac{T}{4} \delta_{i j}
$$

we get the result

$$
\begin{aligned}
& \Delta \omega_{K}\left(\nu_{e}+n \rightarrow e^{-}+p\right)=\frac{G_{F}^{2}\left(C_{V}^{2}+3 C_{A}^{2}\right)}{2 \pi^{3}}\left(\frac{T}{2 M_{n}}\right) \int_{0}^{\infty} d\left|\mathbf{p}^{\prime}\right|\left|\mathbf{p}^{\prime}\right|^{2} q_{0}^{2} \Theta\left(q_{0}\right) F_{\nu}\left(q_{0}\right) \\
& \quad \times\left[1-F_{e}\left(p_{0}^{\prime}\right)\right]\left[-3+\frac{q_{0}^{2}}{T_{\nu}^{2}}\left[1-F_{\nu}\left(q_{0}\right)\right]\left[1-2 F_{\nu}\left(q_{0}\right)\right]-\frac{\left|\mathbf{p}^{\prime}\right|^{2}}{T^{2}} F_{e}\left(p_{0}^{\prime}\right)\left[1-2 F_{e}\left(p_{0}^{\prime}\right)\right]\right. \\
& \left.-3 \frac{q_{0}}{T_{\nu}}\left[1-F_{\nu}\left(q_{0}\right)\right]+3 \frac{p_{0}^{\prime}}{T} F_{e}\left(p_{0}^{\prime}\right)+\left(\frac{C_{A}^{2}-C_{V}^{2}}{C_{V}^{2}+3 C_{A}^{2}}\right)\left(\frac{2\left|\mathbf{p}^{\prime}\right|^{2} q_{0}}{3 T T_{\nu} p_{0}^{\prime}}\right) F_{e}\left(p_{0}^{\prime}\right)\left[1-F_{\nu}\left(q_{0}\right)\right]\right] .
\end{aligned}
$$

The above expression for $\Delta \omega_{K}\left(\nu_{e}+n \rightarrow e^{-}+p\right)$ cannot be extended to all reactions of (1.2) by simply using the substitution rules of Table 1 . This is due to the different form of the statistical factors involved in the corresponding expressions of (4.7). In Appendix $\mathrm{C}$ we have reported the corresponding correction terms for the other processes $(b)-(f)$ in (1.2) . Using these expressions the contribution $\Delta \omega_{K}$ can be numerically evaluated. We report the results in Figure 4.

\section{Thermal radiative corrections}

In the evaluation of the $n \leftrightarrow p$ rates, in addition to the pure electromagnetic radiative corrections, already analyzed in the previous sections, it is also necessary to include thermal radiative corrections [13, 14]. These are due to finite temperature effects in the radiative processes of Figure 5, whose strength is expected to be of the order of $\alpha\left(T / m_{e}\right)$, and are related to the interactions of the in/out particles involved in the microscopic processes $(a)-(f)$ with the surrounding plasma of $e^{+}-e^{-}$pairs, $\nu_{e}-\bar{\nu}_{e}$ and photons. As in previous sections, for seek of brevity we will explicitly illustrate the computation of corrections 
for the particular process $\nu_{e}+n \rightarrow e^{-}+p$. The generalization to all six processes is straightforward using Table 1.

To compute such thermal effects it is useful to apply the standard real time formalism for finite temperature quantum field theory [15]. In this approach propagators for $\gamma$, $e^{ \pm}, \nu_{e}$ and $\bar{\nu}_{e}$ appearing in Feynman graphs of Figure 5, take the following expression, containing a finite density term as well

$$
\begin{aligned}
i \Delta_{\gamma}^{\mu \nu}(k) & =-\left[\frac{i}{k^{2}}+2 \pi \frac{\delta\left(k^{2}\right)}{e^{\beta\left|k_{0}\right|}-1}\right] g^{\mu \nu}=-\left[\frac{i}{k^{2}}+2 \pi \delta\left(k^{2}\right) B\left(k_{0}\right)\right] g^{\mu \nu} \\
i S_{e}\left(p^{\prime}\right) & =\frac{i}{\not p^{\prime}-m_{e}}-2 \pi \delta\left(p^{\prime 2}-m_{e}^{2}\right) F_{e}\left(p_{0}^{\prime}\right)\left(\not p^{\prime}+m_{e}\right) \\
i S_{\nu}(q) & =\frac{i}{\not q}-2 \pi \delta\left(q^{2}\right) F_{\nu}\left(q_{0}\right) \not d
\end{aligned}
$$

The additional contributionsf pick up real particle via the mass-shell $\delta$-functions and are proportional to the particle densities in the thermal bath. They take into account the role played by the medium in the single particle propagation. Note that a similar term for nucleons would be suppressed by a Boltzmann factor smaller than $\exp (-100)$ in the temperature range relevant for $\mathrm{BBN}$, so it will be neglected in the following. Hereafter we will denote with thermal radiative corrections the finite temperature effects for all processes corresponding to the graphs in Figure 5, once the pure radiative contributions, considered in previous sections, have been subtracted.

The diagrams of Figures 5(a), 5(b) provide corrections of order $\alpha\left(T / m_{e}\right)$ via the interference terms with the corresponding tree-level diagram. In particular, the diagram of Figure 5(a) is responsible for electron mass shift and wave function thermal renormalization terms. The diagram of Figure 5(b) provides the vertex correction via its interference with the tree-level process. Finally, in order to cure the infrared/collinear divergences present in the 5(a) and 5(b) contributions, it is also necessary to consider the squared amplitudes corresponding to the diagrams of Figures 5(c)-5(f), which describe photon emission and absorption processes.

\footnotetext{
${ }^{5}$ We remind that we are assuming vanishing chemical potential for all species.
} 


\subsection{Electron mass shift and thermal wave function renormaliza- tion}

As already stated, the diagram of Figure 5(a) is the electron self-energy contribution to the $\nu_{e}+n \rightarrow e^{-}+p$ weak process and contributes to thermal radiative corrections via electron mass and wave function renormalization. The electron propagator reads

$$
\widetilde{S}_{e}=\frac{1}{\not p^{\prime}-m_{e}-\Sigma}
$$

where the self-energy $\Sigma$ is given by

$$
\Sigma\left(p^{\prime}\right)=i 4 \pi \alpha \int \frac{d^{4} k}{(2 \pi)^{4}} \gamma^{\mu} S_{e}\left(p^{\prime}-k\right) \gamma^{\nu} \Delta_{\mu \nu}(k)
$$

Hence, the thermal part of $\Sigma$ is

$$
\Sigma_{T}\left(p^{\prime}\right)=-\frac{\alpha}{\pi^{2}} \int d^{4} k\left[\frac{2 m_{e}-\not p^{\prime}+\not k}{\left(p^{\prime}-k\right)^{2}-m_{e}^{2}} B\left(k_{0}\right) \delta\left(k^{2}\right)-\frac{2 m_{e}+\not k}{\left(p^{\prime}+k\right)^{2}} F_{e}(E) \delta\left(k^{2}-m_{e}^{2}\right)\right] \text {, }
$$

with $E=\sqrt{|\mathbf{k}|^{2}+m_{e}^{2}}$.

By choosing the direction of electron propagation along the 3-axis, the thermal selfenergy $\Sigma_{T}$ takes the simple general form

$$
\Sigma_{T}=\Sigma_{0}+\gamma^{0} \Sigma_{1}+\gamma^{3} \Sigma_{2}
$$

where the explicit expressions of the $\Sigma_{i}$ can be deduced by comparing (5.6) with (5.7).

Differently from the usual self-energy contribution, the finite temperature contribution contained in $\Sigma_{T}$ is not Lorentz invariant. This is due to the presence of the particle densities $B\left(k_{0}\right)$ and $F_{e}(E)$, which take the explicit expressions reported in (5.6) only in the comoving frame. Actually, the lack of Lorentz covariance, due to the presence of the preferred frame of the medium, makes tricky the definition of a particle state, which is generally defined as an irreducible representation of the Poincarè group. All these difficulties originated a long debate in literature [13, 14], [16]-27], concerning the correct way to evaluate the thermal wave-function renormalization, which is strongly affected by this ambiguity. Recently, a simple approach [27] has been developed in which the definition of particle naturally comes out as the energy pole of the perturbed propagator. 
This approach leads to the following expression for the renormalized projector on positive energy states

$$
\Lambda_{R}^{+}=\left(1+\widehat{\Sigma}_{1}^{\prime}+\frac{\left|\mathbf{p}^{\prime}\right|}{p_{0}^{\prime}} \widehat{\Sigma}_{2}^{\prime}+\frac{m_{e}}{p_{0}^{\prime}} \widehat{\Sigma}_{0}^{\prime}\right) \frac{\left(\not p^{\prime}+m_{e}^{R}\right)}{2 p_{0}^{\prime R}}-\frac{1}{2 p_{0}^{\prime}}\left(\frac{\widehat{\Sigma}_{2}}{\left|\mathbf{p}^{\prime}\right|}+\frac{\widehat{\Sigma}_{1}}{p_{0}^{\prime}}\right)\left[\mathbf{p}^{\prime} \cdot \gamma+\frac{\left|\mathbf{p}^{\prime}\right|^{2}}{m_{e}}\right]
$$

In the previous expression $p_{0}^{\prime}=\sqrt{\left|\mathbf{p}^{\prime}\right|^{2}+m_{e}^{2}}, p_{0}^{\prime R}=\sqrt{\left|\mathbf{p}^{\prime}\right|^{2}+\left(m_{e}^{R}\right)^{2}}$. These notations will be implicitly used hereafter. The hat on $\Sigma_{i}$ and $\Sigma_{i}^{\prime} \equiv d \Sigma_{i} / d p_{0}^{\prime}$ reminds that they are evaluated on shell, i.e. for $p_{0}^{\prime}=\sqrt{\left|\mathbf{p}^{\prime}\right|^{2}+m_{e}^{2}}$. Furthermore, the electron renormalized mass $m_{e}^{R}$ results to be

$$
m_{e}^{R}=m_{e}+\widehat{\Sigma}_{0}+\frac{p_{0}^{\prime}}{m_{e}} \widehat{\Sigma}_{1}+\frac{\left|\mathbf{p}^{\prime}\right|}{m_{e}} \widehat{\Sigma}_{2}
$$

A similar result is obtained for projector on negative energy states $\Lambda_{R}^{-}$[27].

As it is clear from (5.8), at finite temperature the projector operator does not renormalize multiplicatively. The presence of an additional term in the expression of $\Lambda_{R}^{ \pm}$was already pointed out in literature [25], but the form of this term did not have the correct limit in the $T=0$ covariant case. This is instead the case of (5.8), as it can be simply verified. However, the additional term in (5.8) does not affect the electron wave-function renormalization contribution to rates for processes (1.2) in the nonrelativistic limit for nucleons, as can be seen by explicit calculation?.

For brevity we only report the expressions] for $\widehat{\Sigma}_{i}$ involved in (5.9)

$$
\begin{aligned}
\widehat{\Sigma}_{0} & =\frac{\alpha m_{e}}{\pi\left|\mathbf{p}^{\prime}\right|} \int_{0}^{\infty} d|\mathbf{k}| \frac{|\mathbf{k}| F_{e}(E)}{E}(\log A-\log B), \\
\widehat{\Sigma}_{1} & =\frac{\pi \alpha T^{2}}{6\left|\mathbf{p}^{\prime}\right|} \log \left(\frac{p_{0}^{\prime}+\left|\mathbf{p}^{\prime}\right|}{p_{0}^{\prime}-\left|\mathbf{p}^{\prime}\right|}\right)+\frac{\alpha}{2 \pi\left|\mathbf{p}^{\prime}\right|} \int_{0}^{\infty} d|\mathbf{k}||\mathbf{k}| F_{e}(E)(\log A+\log B), \\
\widehat{\Sigma}_{2} & =\frac{\pi \alpha T^{2}}{3\left|\mathbf{p}^{\prime}\right|}\left[1-\frac{p_{0}^{\prime}}{2\left|\mathbf{p}^{\prime}\right|} \log \left(\frac{p_{0}^{\prime}+\left|\mathbf{p}^{\prime}\right|}{p_{0}^{\prime}-\left|\mathbf{p}^{\prime}\right|}\right)\right]+\frac{2 \alpha}{\pi\left|\mathbf{p}^{\prime}\right|} \int_{0}^{\infty} d|\mathbf{k}| \frac{|\mathbf{k}|^{2} F_{e}(E)}{E} \\
& \times\left[1-\frac{p_{0}^{\prime} E+m_{e}^{2}}{4|\mathbf{k}|\left|\mathbf{p}^{\prime}\right|} \log A-\frac{p_{0}^{\prime} E-m_{e}^{2}}{4|\mathbf{k}|\left|\mathbf{p}^{\prime}\right|} \log B\right]
\end{aligned}
$$

with

$$
A=\frac{p_{0}^{\prime} E+m_{e}^{2}+|\mathbf{k}|\left|\mathbf{p}^{\prime}\right|}{p_{0}^{\prime} E+m_{e}^{2}-|\mathbf{k}|\left|\mathbf{p}^{\prime}\right|} \quad, \quad B=\frac{p_{0}^{\prime} E-m_{e}^{2}+|\mathbf{k}|\left|\mathbf{p}^{\prime}\right|}{p_{0}^{\prime} E-m_{e}^{2}-|\mathbf{k}|\left|\mathbf{p}^{\prime}\right|} .
$$

\footnotetext{
${ }^{6}$ To evaluate thermal radiative corrections one can safely assume the approximation of infinite mass for nucleons.

${ }^{7}$ We note that our result for $\widehat{\Sigma}_{2}$ differs for a numerical factor from the analogous one of Ref. 14.
} 
For the process $\nu_{e}+n \rightarrow e^{-}+p$ the Born graph yields the expression for the rate for nucleon given by (2.5). The additional contribution due to the electron mass renormalization $\Delta \omega_{M}$ can be obtained by replacing $m_{e} \rightarrow m_{e}^{R}$ in (2.5) ; thus at order $\alpha$ we get

$$
\begin{aligned}
\Delta \omega_{M}\left(\nu_{e}+n \rightarrow\right. & \left.e^{-}+p\right)=\frac{G_{F}^{2}\left(C_{V}^{2}+3 C_{A}^{2}\right)}{2 \pi^{3}} \frac{\alpha}{\pi} \int_{0}^{\infty} d\left|\mathbf{p}^{\prime}\right|\left|\mathbf{p}^{\prime}\right|^{2} q_{0}^{2} \Theta\left(q_{0}\right) F_{\nu}\left(q_{0}\right)\left[1-F_{e}\left(p_{0}^{\prime}\right)\right] \\
& \times\left(\frac{2}{q_{0}}+\frac{F_{e}\left(p_{0}^{\prime}\right)}{T}-\frac{1-F_{\nu}\left(q_{0}\right)}{T_{\nu}}\right)\left\{\frac{\pi^{2} T^{2}}{3 p_{0}^{\prime}}+\frac{2}{p_{0}^{\prime}} \int_{0}^{\infty} d|\mathbf{k}||\mathbf{k}|^{2} \frac{F_{e}(E)}{E}\right. \\
& \left.+\frac{m_{e}^{2}}{2\left|\mathbf{p}^{\prime}\right| p_{0}^{\prime}} \int_{0}^{\infty} d|\mathbf{k}||\mathbf{k}| \frac{F_{e}(E)}{E}(\log A-\log B)\right\}
\end{aligned}
$$

Note that the extension of the above result to all reactions of (1.2) cannot be simply done using the substitution rule of Table 1, since the dependence on the renormalized mass comes through the statistical factors of the Born expression as well, which depend on the particular reaction. In Appendix $\mathrm{C}$ we have reported how (5.14) must be modified to be applied to the other processes $(b)-(f)$.

The contribution due to the electron wave function renormalization is obtained by using the projector (5.8) in the evaluation of the Born rate. This procedure, having subtracted the contribution due to the mass renormalization gives, at order $\alpha$

$$
\begin{aligned}
\Delta \omega_{W}\left(\nu_{e}+n \rightarrow\right. & \left.e^{-}+p\right)=-\frac{G_{F}^{2}\left(C_{V}^{2}+3 C_{A}^{2}\right)}{2 \pi^{3}} \frac{\alpha}{\pi} \int_{0}^{\infty} d\left|\mathbf{p}^{\prime}\right|\left|\mathbf{p}^{\prime}\right|^{2} q_{0}^{2} \Theta\left(q_{0}\right) F_{\nu}\left(q_{0}\right)\left(1-F_{e}\left(p_{0}^{\prime}\right)\right) \\
& \times\left\{\frac{\pi^{2} T^{2}}{6\left|\mathbf{p}^{\prime}\right| p_{0}^{\prime}} \log \left(\frac{p_{0}^{\prime}+\left|\mathbf{p}^{\prime}\right|}{p_{0}^{\prime}-\left|\mathbf{p}^{\prime}\right|}\right)+2 \int_{0}^{\infty} d|\mathbf{k}||\mathbf{k}|^{2} \frac{F_{e}(E)}{E} \frac{1}{\left|\mathbf{p}^{\prime}\right|^{2}-|\mathbf{k}|^{2}}\right. \\
& \left.+\frac{1}{2\left|\mathbf{p}^{\prime}\right| p_{0}^{\prime}} \int_{0}^{\infty} d|\mathbf{k}||\mathbf{k}| \frac{F_{e}(E)}{E}\left[\left(E+p_{0}^{\prime}\right) \log A+\left(E-p_{0}^{\prime}\right) \log B\right]\right\}
\end{aligned}
$$

In the expression (5.15) we have also subtracted an infrared divergent term which is cancelled by an opposite term provided by the photon absorption and emission rate. The collinear divergence, still present in (5.15) for $\left|\mathbf{p}^{\prime}\right|=|\mathbf{k}|$, together with a similar contribution coming from the vertex correction is also compensated by bremsstrählung diagrams.

Differently from (5.14), the extension of (5.15) to other processes can be straightforwardly obtained by using Table 1 . The results for $\Delta \omega_{M}$ and $\Delta \omega_{W}$, normalized to $(\alpha / \pi) \omega_{B}$ versus photon temperature are reported in Figures 6 and 7, respectively. 


\subsection{Vertex Corrections}

The vertex correction is provided, at order $\alpha$, by the interference term between the diagram of Figure 5(b) and the tree amplitude of Figure 1, leading to 9

$$
\begin{aligned}
& \Delta \omega_{V}\left(\nu_{e}+n \rightarrow e^{-}+p\right)=\frac{G_{F}^{2}\left(C_{V}^{2}+3 C_{A}^{2}\right)}{2 \pi^{3}} \frac{\alpha}{\pi} \int_{0}^{\infty} d\left|\mathbf{p}^{\prime}\right|\left|\mathbf{p}^{\prime}\right| \int_{0}^{\infty} d|\mathbf{k}||\mathbf{k}| q_{0}^{2} \Theta\left(q_{0}\right) \\
& \quad \times F_{\nu}\left(q_{0}\right)\left[1-F_{e}\left(p_{0}^{\prime}\right)\right] \frac{F_{e}(E)}{E}\left\{\frac{E}{p_{0}^{\prime}+E} \log A+\frac{E}{p_{0}^{\prime}-E} \log B-\frac{2|\mathbf{k}|\left|\mathbf{p}^{\prime}\right|}{\left|\mathbf{p}^{\prime}\right|^{2}-|\mathbf{k}|^{2}}\right\} .
\end{aligned}
$$

The expression (5.16) is extended to the other processes by using Table 1. We have reported the results in Figure 8.

\subsection{Photon Emission and Absorption}

In order to eliminate the infrared divergences due to the radiative diagrams of Figures $5(\mathrm{a})$ and $5(\mathrm{~b})$, it is necessary to include the rates of processes in which a photon is either absorbed or emitted. In fact, they exactly provide the divergent contributions which cancel the corresponding ones due to vertex and wave function renormalizations. The processes give a finite contribution as well, which takes the following form

$$
\begin{array}{r}
\Delta \omega_{\gamma}\left(\nu_{e}+n \rightarrow e^{-}+p\right)=\frac{G_{F}^{2}\left(C_{V}^{2}+3 C_{A}^{2}\right)}{2 \pi^{3}} \frac{\alpha}{\pi} \int_{0}^{\infty} d\left|\mathbf{p}^{\prime}\right| \int_{0}^{\infty} d|\mathbf{k}| \frac{\left|\mathbf{p}^{\prime}\right|^{2}}{p_{0}^{\prime}} B(|\mathbf{k}|) \\
\times\left[1-F_{e}\left(p_{0}^{\prime}\right)\right]\left\{-\left[\frac{2 p_{0}^{\prime}}{|\mathbf{k}|}-\frac{p_{0}^{\prime 2}}{|\mathbf{k}|\left|\mathbf{p}^{\prime}\right|} \log \left(\frac{p_{0}^{\prime}+\left|\mathbf{p}^{\prime}\right|}{p_{0}^{\prime}-\left|\mathbf{p}^{\prime}\right|}\right)\right]\left[\widetilde{Q}_{+}^{2}+\widetilde{Q}_{-}^{2}-2 \widetilde{Q}^{2}\right]\right. \\
\left.-\left[2 S-\frac{p_{0}^{\prime}}{\left|\mathbf{p}^{\prime}\right|} \log \left(\frac{p_{0}^{\prime}+\left|\mathbf{p}^{\prime}\right|}{p_{0}^{\prime}-\left|\mathbf{p}^{\prime}\right|}\right)\right]\left[\widetilde{Q}_{+}^{2}-\widetilde{Q}_{-}^{2}\right]+\frac{|\mathbf{k}|}{2\left|\mathbf{p}^{\prime}\right|} \log \left(\frac{p_{0}^{\prime}+\left|\mathbf{p}^{\prime}\right|}{p_{0}^{\prime}-\left|\mathbf{p}^{\prime}\right|}\right)\left[\widetilde{Q}_{+}^{2}+\widetilde{Q}_{-}^{2}\right]\right\},
\end{array}
$$

where $\widetilde{Q}_{ \pm}^{2} \equiv\left(q_{0} \pm|\mathbf{k}|\right)^{2} F_{\nu}\left(q_{0} \pm|\mathbf{k}|\right) \Theta\left(q_{0} \pm|\mathbf{k}|\right), \widetilde{Q}^{2} \equiv q_{0}^{2} F_{\nu}\left(q_{0}\right) \Theta\left(q_{0}\right)$ and $S=1$. The expression (5.17) is extended to the other processes by using Table 1 and with $S=1$ for $\nu_{e}+n \leftrightarrow e^{-}+p$ and $n \leftrightarrow e^{-}+\bar{\nu}_{e}+p$, and $S=0$ for $e^{+}+n \leftrightarrow \bar{\nu}_{e}+p$. The ratio $\Delta \omega_{\gamma} /(\alpha / \pi) \omega_{B}$ is plotted for all processes (1.2) in Figure 9. The results agree with the ones reported in Figure 8 of Ref. [14 apart from the channel $e^{-}+p \rightarrow \bar{\nu}_{e}+n$, which is there underestimated by a factor 10 . Notice that $\Delta \omega_{\gamma}$ represents the leading thermal correction to neutron decay and the inverse reaction, in the temperature range relevant for BBN. This is simply understood since the inclusion of the process $\gamma+n \rightarrow e^{-}+\bar{\nu}_{e}+p$ greatly increases the neutron decay rates, otherwise strongly suppressed by phase space.

\footnotetext{
${ }^{8}$ The result for $\Delta \omega_{V}$ quoted in [14] as Eq. (11) has a missing factor $1 / E$.
}

${ }^{9}$ Our result slightly differs from Eq. (13) in Ref. [14]. 


\section{Analysis of the results and ${ }^{4} \mathrm{He}$ abundance}

In the previous sections we have reported a detailed analysis of three kind of corrections to Born rates for $n \leftrightarrow p$ processes: zero temperature radiative corrections, finite nucleon mass and thermal radiative effects. With reference to Figures (3), (4) and (6)-(9) the results for each reaction channel can be summarized as follows.

(a) $\nu_{e}+n \rightarrow e^{-}+p$

For the crucial BBN temperature range, $0.1 \mathrm{MeV} \leq T \leq 3.5 \mathrm{MeV}$, the two main corrections to the Born rate come from zero temperature radiative and kinetic terms. The contribution $\Delta \omega_{R}$ is weakly depending on $T$ and represents the dominant term, though for large temperature the kinetic correction $\Delta \omega_{K}$ starts contributing significantly. The two combined contributions correct the Born rate for a factor $6 \div 9 \%$, whilst thermal radiative ones are of the order of $1 \%$.

(b) $e^{-}+p \rightarrow \nu_{e}+n$

For this channel radiative corrections are dominant at low temperature, while the kinetic ones give a quite relevant effect in whole BBN temperature range and correct the Born rate for a factor varying from $1 \%$ at low $T$ up to $3 \%$ for $T=3 \div 4 \mathrm{MeV}$. The radiative contribution is quite rapidly decreasing with temperature, reaching large negative values. This leads to a partial cancellation between $\Delta \omega_{R}$ and $\Delta \omega_{K}$. Thermal corrections are dominated by bremsstrählung contribution $\Delta \omega_{\gamma}$ and can be as large as $2 \%$ of $\omega_{B}$ for large temperature.

(c) $e^{+}+n \rightarrow \bar{\nu}_{e}+p$

For this process the radiative corrections have a behaviour quite similar to channel $(b)$, though they are even more rapidly decreasing with temperature. This again leads to a partial cancellation between $\Delta \omega_{R}$ and the positive monotonically increasing $\Delta \omega_{K}$. Thermal corrections are again mainly provided by photon emission/absorption and monotonically increase with temperature up to a factor $2 \%$ of the corresponding Born rate. 
(d) $\bar{\nu}_{e}+p \rightarrow e^{+}+n$

The radiative and kinetic corrections sum up to a factor of about $5 \%$ of the Born rate. Actually the opposite behaviour of $\Delta \omega_{R}$ and $\Delta \omega_{K}$ conspires to give an almost constant total correction in the whole interesting temperature range. Thermal effects are quite small, contributing for less than $0.5 \%$.

(e) $n \rightarrow e^{-}+\bar{\nu}_{e}+p$

For the neutron decay the radiative corrections are practically constant and give the leading effect for small temperature, $T \leq m_{e}$. For larger $T$ the thermal photon emission/absorption processes rapidly become dominating over all other correction terms with a relative ratio to the Born rate as large as $10^{4}$. However, this large correction is weakly contributing to total $n \rightarrow p$ rate, and so to ${ }^{4} \mathrm{He}$ abundance prediction, since in the temperature range $T>m_{e}$ the scattering processes $(a)-(d)$ are largely dominant over decay and inverse decay.

(f) $e^{-}+\bar{\nu}_{e}+p \rightarrow n$

Same considerations of the direct process $(e)$ hold for inverse neutron decay. As for the direct channel, the kinetic contribution is negative down to temperatures of the order $T \sim 0.2 \mathrm{MeV}$. The contribution of $\left|\Delta \omega_{K}\right|$ is however negligible for both processes, smaller than $1 \%$.

All results are summarized in Figure 10, where we have shown the total relative corrections in percent. For the processes $(a)$ and $(d)$ the correction is almost constant over the entire considered range for $T$, and of the order of $6 \div 10 \%$ and $5 \div 6 \%$, respectively. The positive kinetic contribution soften the deep decreasing of the radiative terms for channels $(b)$ and $(c)$. Finally the large effect of thermal bremsstrählung for neutron decay and inverse process $(e)$ and $(f)$ is particularly evident.

We have shown the total rates for $n \leftrightarrow p$ in Figure 11, while the total relative correction $\Delta \omega / \omega_{B} \equiv\left(\omega-\omega_{B}\right) / \omega_{B}$, in percent, are plotted in Figure 12. For future applications in the $\mathrm{BBN}$ codes we have performed a fit of the numerical results for $\omega(n \rightarrow p)$ and $\omega(p \rightarrow n)$. 
The fitting expressions are the following

$$
\begin{aligned}
\omega(n \rightarrow p) & =\frac{1}{\tau_{n}^{\text {exp }}} \sum_{l=0}^{8} a_{l}\left(\frac{T}{m_{e}}\right)^{l}, \\
\omega(p \rightarrow n) & =\frac{1}{\tau_{n}^{e x p}} \exp \left(-\frac{q m_{e}}{T}\right) \sum_{l=1}^{10} b_{l}\left(\frac{T}{m_{e}}\right)^{l},
\end{aligned}
$$

where for $n \rightarrow p$

$$
\begin{gathered}
\quad a_{0}=1 ; \quad a_{1}=1.0988 ; \quad a_{2}=-9.8297 ; a_{3}=17.379 ; a_{4}=32.197 ; \\
a_{5}=27.372 ; \quad a_{6}=-0.20975 ; \quad a_{7}=0.67033 \cdot 10^{-2} ; a_{8}=-0.91503 \cdot 10^{-4}
\end{gathered}
$$

while for $p \rightarrow n$

$$
\begin{gathered}
b_{1}=22.138 ; \quad b_{2}=-77.737 ; \quad b_{3}=130.76 ; b_{4}=-22.010 ; \quad b_{5}=50.262 ; \\
b_{6}=-4.8032 ; \quad b_{7}=0.53087 ; b_{8}=-0.03403 ; b_{9}=0.11508 \cdot 10^{-2} ; \\
b_{10}=-0.15850 \cdot 10^{-4} ; q=2.9123 .
\end{gathered}
$$

The fit has been obtained requiring that the fitting functions differ by less than $0.1 \%$ from the numerical values.

The whole correction $\Delta \omega$ for $n \leftrightarrow p$ turn out to be a positive decreasing function over the whole temperature range relevant for BBN. The main contribution at low temperature for both total rates comes from the radiative corrections, while for $T>2 \div 3 \mathrm{MeV}$ kinetic contribution starts dominating. This is particularly evident by looking at Figures 13 and 14, where $\Delta \omega_{R} / \omega_{B}$ and $\Delta \omega_{K} / \omega_{B}$ are separately plotted. While for radiative corrections the effect on $n \rightarrow p$ total rate is larger than on the $p \rightarrow n$ one, $\Delta \omega_{K}$ shows an opposite behaviour. The competition of these two corrections is then responsible for the presence of the inversion point in Figure 12 at $T \simeq 0.15 \mathrm{MeV}$. Finally, the pure thermal radiative corrections

$$
\Delta \omega_{T} \equiv \Delta \omega_{M}+\Delta \omega_{W}+\Delta \omega_{V}+\Delta \omega_{\gamma}
$$

are plotted in Figure 15. Their order of magnitude is sensibly smaller, but they nevertheless may contribute for a factor $0.2 \div 0.4 \%$ at the freeze-out temperature $T \sim 1 \mathrm{MeV}$. Although some differences in the expressions for several contributions, our result for $\Delta \omega_{T}$ essentially agree with the results of Ref.s [13, 14]. 
The corrections on the $n \leftrightarrow p$ rates, as well as the plasma electron mass correction to neutrino temperature, considered in Appendix A, may produce a sensible correction to the ${ }^{4} \mathrm{He}$ mass fraction $Y_{p}$, which is strongly dependent on the neutron fraction $X_{n}=$ $n_{n} /\left(n_{n}+n_{p}\right)$ at the nucleon freeze-out. An accurate theoretical prediction for Helium abundance, as well as for the other light nuclei produced during BBN, can be obtained by using the standard BBN code [7] suitably modified to take into account all the above corrections [32]. Here we report the results for the expected variation of the surviving neutron fraction $X_{n}$ induced by the whole effects $\Delta \omega(n \leftrightarrow p)$. This allows for a simple estimate of the corresponding variation of $Y_{p}$.

The neutron fraction is determined by the differential equation

$$
\frac{d X_{n}}{d T}=\frac{d t}{d T}\left[\omega(p \rightarrow n)\left(1-X_{n}\right)-\omega(n \rightarrow p) X_{n}\right] .
$$

Writing $X_{n}=X_{n}^{0}+\delta X_{n}$, where $\delta X_{n}$ is the correction induced by $\Delta \omega$, we have at first order

$$
\begin{aligned}
\frac{d X_{n}^{0}}{d T} & =\frac{d t}{d T}\left[\omega_{B}(p \rightarrow n)\left(1-X_{n}^{0}\right)-\omega_{B}(n \rightarrow p) X_{n}^{0}\right], \\
\frac{d}{d T} \delta X_{n} & =-\frac{d t}{d T}\left[\left(\omega_{B}(p \rightarrow n)+\omega_{B}(n \rightarrow p)\right) \delta X_{n}-\Delta \omega(p \rightarrow n)\right. \\
& \left.+(\Delta \omega(p \rightarrow n)+\Delta \omega(n \rightarrow p)) X_{n}^{0}\right] .
\end{aligned}
$$

Notice that the zero order abundance $X_{n}^{0}$ has been defined as the one obtained by the Born amplitudes rescaled by the constant factor 961/886.7, which provides at tree level the correct prediction for neutron lifetime (see our discussion in section 3). The Born rates $\omega_{B}(p \leftrightarrow n)$ in (6.7) are therefore rescaled by the same factor. Equations (6.7) have been numerically solved using our fitting function for $\omega(n \leftrightarrow p)$ and a similar one for $\omega_{B}(n \leftrightarrow p)$, which we do not report for brevity. We found for the asymptotic abundance $\delta X_{n} \simeq 0.0024$, with a relative change, in percent, $\delta X_{n} / X_{n}^{0}=1.6 \%$. It is also interesting

to evaluate the effect due to the thermal radiative contribution. Using again (6.7), with now the $\Delta \omega_{T}(n \leftrightarrow p)$ in the r.h.s., we get $\delta X_{n}^{T}=-0.0002, \delta X_{n}^{T} / X_{n}^{0}=-0.15 \%$.

These results allows for a simple estimation of corrections to ${ }^{4} \mathrm{He}$ mass fraction 33

$$
\delta Y_{p}=2 \delta X_{n} \exp \left(-\frac{t_{n s}}{\tau_{n}^{e x p}}\right)
$$


where $t_{n s} \simeq 180 \mathrm{sec}$ corresponds to the onset of nucleosynthesis and the exponential factor accounts for the depletion of relic neutrons at freeze-out due to $\beta$-decay. Using the results for $\delta X_{n}$ we find

$$
\begin{array}{lll}
\delta Y_{p} \simeq-0.0004, & \frac{\delta Y_{p}}{Y_{p}}=-0.15 \%, & \text { thermal radiative } \\
\delta Y_{p} \simeq 0.004, \quad \frac{\delta Y_{p}}{Y_{p}}=1.6 \%, & \text { total }
\end{array}
$$

The thermal radiative corrections decrease the value of $Y_{p}$, in agreement with the results

of [13] and [14]. However, the total correction is largely dominated by zero temperature radiative and finite mass corrections, which give a positive contribution to $Y_{p}$. The total correction, evaluated with the above method, agrees with the recent result obtained in [34. They also include the effect of finite temperature QED corrections to the equation of state of the electromagnetic plasma [35] and of residual coupling of neutrinos to the thermal bath during the $e^{+}-e^{-}$annihilation phase [13], [28]. However, the two combined corrections give a very small positive contribution, of the order $\delta Y_{p} \simeq(2 \div 3) \cdot 10^{-4}$.

\section{Conclusions and Outlook}

In this paper we have performed a complete analysis of the corrections to the tree level rates (1.2) . These reactions are relevant to fix the neutron density at the freeze-out, which is the first step of the primordial synthesis of light nuclei. In particular we have considered order $\alpha$ radiative corrections, $1 / M_{N}$ effects obtained relaxing the assumption of infinite massive nucleons, and thermal radiative effects on the rates as well as on the behaviour of neutrino temperature after decoupling. The total correction $\Delta \omega$ to the rate per nucleon is a monotonically decreasing function of the temperature in the interval $0.1 \mathrm{MeV} \leq T \leq 3.5 \mathrm{MeV}$, for both processes $n \leftrightarrow p$. It varies in the range $(2 \div 8) \%$ of the Born rates. The main effects come from radiative and kinematical terms $\Delta \omega_{R}$ and $\Delta \omega_{K}$, considered in section 3 and 4 , respectively. We find that $\Delta \omega_{R}$ contributes for about $(-1 \div 8) \%$ in the above range for $T$, while $\Delta \omega_{K}$, which originates from the relative motion of the initial nucleon with respect to the comoving frame, grows with temperature up to a value of about $2.5 \%$ of $\omega_{B}(n \leftrightarrow p)$. Thermal radiative corrections $\Delta \omega_{T}$, evaluated in the real time formalism, rapidly increase with temperature and only contribute for about 
$(0.1 \div 0.7) \%$

We have calculated how these corrections affect the theoretical prediction for ${ }^{4} \mathrm{He}$ mass fraction $Y_{p}$. We have solved the differential equation governing the evolution of neutron fraction $X_{n}$ and found for the total correction induced on $Y_{p}$ by $\Delta \omega$ the value $\delta Y_{p} \simeq 0.004$, which corresponds to a relative effect $\delta Y_{p} / Y_{p} \simeq 1.6 \%$. This result has been obtained using the simple method outlined in [33]. The thermal radiative effects alone contribute to this variation for a negative factor $\delta Y_{p}=-0.0004$, in agreement with the results of [13] and [14]. Finally, the total correction to ${ }^{4} \mathrm{He}$ abundance is in substantial agreement with a recent estimate [34], though the result for the thermal radiative small correction is of the same magnitude but with an opposite sign. A more careful analysis of the effect of $\Delta \omega$ on Helium and all other nuclei abundances, using a modification of the BBN codes [7], is in progress [32].

\section{Acknowledgements}

We thank E.W. Kolb for stimulating our interest to the subject considered in this paper and for a useful discussion. 


\section{A Neutrino temperature versus photon temperature}

In this Appendix we evaluate the ratio of neutrino temperature $T_{\nu}$ over the photon temperature $T$.

When weak interactions go out of equilibrium, at $T \sim 1 \mathrm{MeV}$, neutrinos decouple from the photon and $e^{+}-e^{-}$thermal bath. Their temperature then decreases as $a(t)^{-1}$, with $a(t)$ the cosmological scale factor. Shortly after, as $T$ drops below the electron mass, electrons and positrons annihilate and transfer their entropy to photons only. To evaluate the ratio $T_{\nu} / T$ we recall the expressions for the entropy density of a particle specie $i$, with $g_{i}$ internal degrees of freedom [2]

$s_{i}=\frac{g_{i}}{6 \pi^{2}} T_{i}^{3} \mathcal{I}\left(x_{i}\right)=\frac{g_{i}}{6 \pi^{2}} T_{i}^{3} \int_{0}^{\infty}\left(y^{2}+2 y x_{i}\right)^{1 / 2}\left(4 y^{2}+8 y x_{i}+3 x_{i}^{2}\right)\left(\exp \left(x_{i}+y\right) \pm 1\right)^{-1} d y$

where,+- refers to fermions and bosons, respectively, and $x_{i}=m_{i} / T_{i}$. Using the fact that the entropy per comoving volume is constant, and in particular that neutrino entropy remains unchanged after decoupling one gets

$$
\frac{T_{\nu}}{T}=\left(\frac{2 \pi^{4}+15 \mathcal{I}\left(x_{e}\right)}{2 \pi^{4}+15 \mathcal{I}\left(x_{e}^{D}\right)}\right)^{1 / 3}
$$

with

$$
x_{e}=\frac{1}{T}\left(m_{e}+\frac{\alpha T^{2}}{m_{e}}\right) \quad ; \quad x_{e}^{D}=\frac{1}{T_{D}}\left(m_{e}+\frac{\alpha T_{D}^{2}}{m_{e}}\right) .
$$

The electron neutrino decoupling temperature, evaluated in [36], is $T_{D} \simeq 2.3 \mathrm{MeV}$. We have verified, that changing $T_{D}$ in the range $2 \div 3 \mathrm{MeV}$ the ratio $T_{\nu} / T$ only changes for a factor less than $0.2 \%$. We have also included the effect of thermal electron mass on neutrino temperature via an effective simple estimate of the form $\alpha T^{2} / m_{e}$ [13].

\section{B Finite nucleon mass corrections to weak ampli- tudes}

In this Appendix we compute the spin-summed squared amplitudes for processes (1.2) for finite nucleon masses.

Let us consider the most general four-fermion effective interaction, responsible for the 
process $p_{1}+p_{2} \rightarrow p_{3}+p_{4}$

$$
M=\frac{G_{F}}{\sqrt{2}} \bar{u}\left(p_{4}\right) O_{\mu} u\left(p_{2}\right) \bar{u}\left(p_{3}\right) \gamma^{\mu}\left(1-\gamma_{5}\right) u\left(p_{1}\right)
$$

where the effective weak coupling $O_{\mu}$ is given by

$$
O_{\mu}=\gamma_{\mu}\left(C_{V}-C_{A} \gamma_{5}\right)+i \frac{f_{2}}{M_{N}} \sigma_{\mu \nu}\left(p_{4}-p_{2}\right)^{\nu}
$$

with $\sigma_{\mu \nu}=(i / 2)\left[\gamma_{\mu}, \gamma_{\nu}\right]$. Adopting the same notation of section 2 we obtain

$$
\begin{aligned}
& \sum_{\text {spins }}|M|^{2} \simeq 8\left(C _ { A } ^ { 2 } \left(m_{1}^{2} m_{2}^{2}+2 m_{1}^{2} m_{3}^{2}+m_{2}^{2} m_{3}^{2}+2 m_{1}^{2} m_{2} m_{4}+2 m_{2} m_{3}^{2} m_{4}\right.\right. \\
& +m_{1}^{2} m_{4}^{2}+2 m_{2}^{2} m_{4}^{2}+m_{3}^{2} m_{4}^{2}-2 m_{1}^{2} s-2 m_{2}^{2} s-2 m_{3}^{2} s-2 m_{4}^{2} s \\
& \left.+2 s^{2}-m_{1}^{2} t-m_{2}^{2} t-m_{3}^{2} t-2 m_{2} m_{4} t-m_{4}^{2} t+2 s t+t^{2}\right) \\
& +C_{V}^{2}\left(m_{1}^{2} m_{2}^{2}+2 m_{1}^{2} m_{3}^{2}+m_{2}^{2} m_{3}^{2}-2 m_{1}^{2} m_{2} m_{4}-2 m_{2} m_{3}^{2} m_{4}\right. \\
& +m_{1}^{2} m_{4}^{2}+2 m_{2}^{2} m_{4}^{2}+m_{3}^{2} m_{4}^{2}-2 m_{1}^{2} s-2 m_{2}^{2} s-2 m_{3}^{2} s-2 m_{4}^{2} s \\
& \left.+2 s^{2}-m_{1}^{2} t-m_{2}^{2} t-m_{3}^{2} t+2 m_{2} m_{4} t-m_{4}^{2} t+2 s t+t^{2}\right) \\
& -2 C_{A} \frac{f_{2}}{M_{N}}\left(m_{1}^{2} m_{2}^{3}-m_{2}^{3} m_{3}^{2}+m_{1}^{2} m_{2}^{2} m_{4}-m_{2}^{2} m_{3}^{2} m_{4}-m_{1}^{2} m_{2} m_{4}^{2}\right. \\
& +m_{2} m_{3}^{2} m_{4}^{2}-m_{1}^{2} m_{4}^{3}+m_{3}^{2} m_{4}^{3}-m_{1}^{2} m_{2} t-m_{2}^{3} t \\
& \text { - } m_{2} m_{3}^{2} t-m_{1}^{2} m_{4} t-m_{2}^{2} m_{4} t-m_{3}^{2} m_{4} t-m_{2} m_{4}^{2} t \\
& \left.-m_{4}^{3} t+2 m_{2} s t+2 m_{4} s t+m_{2} t^{2}+m_{4} t^{2}\right) \\
& +2 C_{V} C_{A}\left(-m_{1}^{2} m_{2}^{2}+m_{2}^{2} m_{3}^{2}+m_{1}^{2} m_{4}^{2}-m_{3}^{2} m_{4}^{2}+m_{1}^{2} t\right. \\
& \left.+m_{2}^{2} t+m_{3}^{2} t+m_{4}^{2} t-2 s t-t^{2}\right) \\
& +2 C_{V} \frac{f_{2}}{M_{N}}\left(m_{1}^{2} m_{2}^{3}+m_{1}^{2} m_{2} m_{3}^{2}-m_{2} m_{3}^{4}-m_{1}^{4} m_{4}-m_{1}^{2} m_{2}^{2} m_{4}\right. \\
& +m_{1}^{2} m_{3}^{2} m_{4}-m_{2} m_{3}^{2} m_{4}^{2}+m_{3}^{2} m_{4}^{3}-m_{1}^{2} m_{2} s+m_{2} m_{3}^{2} s \\
& +m_{1}^{2} m_{4} s-m_{3}^{2} m_{4} s-m_{1}^{2} m_{2} t-m_{2}^{3} t+m_{2}^{2} m_{4} t \\
& \text { - } \left.\left.m_{3}^{2} m_{4} t+m_{2} m_{4}^{2} t-m_{4}^{3} t+m_{2} t^{2}+m_{4} t^{2}\right)\right) \text {. }
\end{aligned}
$$

In the above formula we have used the following definition of the two independent Mandelstam invariants

$$
\begin{aligned}
s & \equiv\left(p_{1}+p_{2}\right)^{2}=\left(p_{3}+p_{4}\right)^{2} \\
t & \equiv\left(p_{1}-p_{3}\right)^{2}=\left(p_{2}-p_{4}\right)^{2}
\end{aligned}
$$


In the formula $(\overline{B .3})$ we have retained only the leading terms, up to first order in the coupling $f_{2}$.

For the process $\nu_{e}+n \rightarrow e^{-}+p$, the corresponding $\sum_{\text {spins }}|M|^{2}$ is obtained from (B.3) by replacing $m_{1}, m_{2}, m_{3}$ and $m_{4}$ with $0, M_{n}, m_{e}$ and $M_{p}$, respectively. Furthermore, from the definition of the Mandelstam invariants and using the four-momentum conservation we have in the neutron rest frame

$$
\begin{aligned}
& s=M_{n}^{2}+2 M_{n} q_{0}, \\
& t=M_{p}^{2}-M_{n}^{2}+2 M_{n}\left(p_{0}^{\prime}-q_{0}\right) .
\end{aligned}
$$

By using (B.3) and changing accordingly the above correspondences one easily obtains the expression for $\sum_{\text {spins }}|M|^{2}$ for all other processes in (1.2) .

\section{Kinematical and thermal mass shift corrections to the rates}

In this Appendix we generalize to all other processes in (1.2) the results reported in (4.17) and (5.14) for kinematical and thermal mass corrections to Born rates for $\nu_{e}+n \rightarrow e^{-}+p$.

The kinematical corrections of section 4.2 can be obtained from (4.17) by using the substitution rule of Table 1 and replacing the factor in square brackets as follows. First of all, note that for the reaction $\bar{\nu}_{e}+p \rightarrow e^{+}+n$ this factor is the same as in (4.17). Differently, for $e^{-}+p \rightarrow \nu_{e}+n$ and $e^{+}+n \rightarrow \bar{\nu}_{e}+p$, one has

$$
\begin{gathered}
{\left[-3-\frac{q_{0}^{2}}{T_{\nu}^{2}} F_{\nu}\left(q_{0}\right)\left[1-2 F_{\nu}\left(q_{0}\right)\right]+\frac{\left|\mathbf{p}^{\prime}\right|^{2}}{T^{2}}\left[1-F_{e}\left(p_{0}^{\prime}\right)\right]\left[1-2 F_{e}\left(p_{0}^{\prime}\right)\right]+3 \frac{q_{0}}{T_{\nu}} F_{\nu}\left(q_{0}\right)\right.} \\
\left.-3 \frac{p_{0}^{\prime}}{T}\left[1-F_{e}\left(p_{0}^{\prime}\right)\right]+\left(\frac{C_{A}^{2}-C_{V}^{2}}{C_{V}^{2}+3 C_{A}^{2}}\right)\left(\frac{2\left|\mathbf{p}^{\prime}\right|^{2} q_{0}}{3 T T_{\nu} p_{0}^{\prime}}\right) F_{\nu}\left(q_{0}\right)\left[1-F_{e}\left(p_{0}^{\prime}\right)\right]\right]
\end{gathered}
$$

while for $n \rightarrow e^{-}+\bar{\nu}_{e}+p$

$$
\begin{aligned}
& {\left[-3-\frac{q_{0}^{2}}{T_{\nu}^{2}} F_{\nu}\left(q_{0}\right)\left[1-2 F_{\nu}\left(q_{0}\right)\right]-\frac{\left|\mathbf{p}^{\prime}\right|^{2}}{T^{2}} F_{e}\left(p_{0}^{\prime}\right)\left[1-2 F_{e}\left(p_{0}^{\prime}\right)\right]+3 \frac{q_{0}}{T_{\nu}} F_{\nu}\left(q_{0}\right)\right.} \\
+ & \left.3 \frac{p_{0}^{\prime}}{T} F_{e}\left(p_{0}^{\prime}\right)-\left(\frac{C_{A}^{2}-C_{V}^{2}}{C_{V}^{2}+3 C_{A}^{2}}\right)\left(\frac{2\left|\mathbf{p}^{\prime}\right|^{2} q_{0}}{3 T T_{\nu} p_{0}^{\prime}}\right) F_{e}\left(p_{0}^{\prime}\right) F_{\nu}\left(q_{0}\right)\right] .
\end{aligned}
$$

Finally for $e^{-}+\bar{\nu}_{e}+p \rightarrow n$

$$
\left[-3+\frac{q_{0}^{2}}{T_{\nu}^{2}}\left[1-F_{\nu}\left(q_{0}\right)\right]\left[1-2 F_{\nu}\left(q_{0}\right)\right]+\frac{\left|\mathbf{p}^{\prime}\right|^{2}}{T^{2}}\left[1-F_{e}\left(p_{0}^{\prime}\right)\right]\left[1-2 F_{e}\left(p_{0}^{\prime}\right)\right]-3 \frac{q_{0}}{T_{\nu}}\left[1-F_{\nu}\left(q_{0}\right)\right]\right.
$$




$$
\left.-3 \frac{p_{0}^{\prime}}{T}\left[1-F_{e}\left(p_{0}^{\prime}\right)\right]-\left(\frac{C_{A}^{2}-C_{V}^{2}}{C_{V}^{2}+3 C_{A}^{2}}\right)\left(\frac{2\left|\mathbf{p}^{\prime}\right|^{2} q_{0}}{3 T T_{\nu} p_{0}^{\prime}}\right)\left[1-F_{e}\left(p_{0}^{\prime}\right)\right]\left[1-F_{\nu}\left(q_{0}\right)\right]\right] .
$$

A similar replacement is required to generalize the result of (5.14). In particular, in addition to the substitutions of Table 1, the factor in (5.14)

$$
\left(\frac{2}{q_{0}}+\frac{F_{e}\left(p_{0}^{\prime}\right)}{T}-\frac{1-F_{\nu}\left(q_{0}\right)}{T_{\nu}}\right)
$$

should be substituted in the following way. As for kinematical corrections, this factor applies to the reaction $\bar{\nu}_{e}+p \rightarrow e^{+}+n$ as well. For $e^{-}+p \rightarrow \nu_{e}+n$ and $e^{+}+n \rightarrow \bar{\nu}_{e}+p$, one has instead

$$
\left(\frac{2}{q_{0}}-\frac{1-F_{e}\left(p_{0}^{\prime}\right)}{T}+\frac{F_{\nu}\left(q_{0}\right)}{T_{\nu}}\right)
$$

while for $n \rightarrow e^{-}+\bar{\nu}_{e}+p$

$$
\left(-\frac{2}{q_{0}}+\frac{F_{e}\left(p_{0}^{\prime}\right)}{T}-\frac{F_{\nu}\left(q_{0}\right)}{T_{\nu}}\right)
$$

and $e^{-}+\bar{\nu}_{e}+p \rightarrow n$

$$
\left(-\frac{2}{q_{0}}-\frac{1-F_{e}\left(p_{0}^{\prime}\right)}{T}+\frac{1-F_{\nu}\left(q_{0}\right)}{T_{\nu}}\right)
$$

\section{References}

[1] P.J.E. Peebles, Physical Cosmology, (Princeton University Press, 1971, Princeton);

S. Weinberg, Gravitation and Cosmology, (Wiley, 1972, New York);

D.N. Schramm and R.V. Wagoner, Ann. Rev. Nucl. Sci. 27 (1977) 37.

[2] E.W. Kolb and M.S. Turner, The Early Universe, (Addison-Wesley Publishing Company, 1990, New York).

[3] K.A. Olive, Proc. of the Workshop TAUP97, Gran Sasso Laboratory, Italy, september 7-11, 1997, astro-ph/9712160;

G. Steigman, astro-ph/9803055.

[4] K.A. Olive and D. Thomas, Astropart. Phys. 7 (1997) 27;

Y.I. Izotov, T.X. Thuan, and V.A. Lipovetsky, Ap. J. Suppl. 108 (1997) 1;

R.F. Carswell, M. Rauch, R.J. Weymann, A.J. Cooke and J.K. Webb, MNRAS 268 
(1994) L1;

A. Songaila, L.L. Cowie, C. Hogan and M. Rugers, Nature 368 (1994) 599;

S. Burles and D. Tytler, Proc. of the ISSI Workshop Primordial Nuclei and their Galactic Evolution, astro-ph/9712265.

[5] S. Sarkar, Rept. Prog. Phys. 59 (1996) 1493.

[6] L.M. Krauss and P. Romanelli, Ap. J. 358 (1990) 47;

P.J. Kernan and L.M. Krauss, Phys. Rev. Lett. 72 (1994) 3309;

G. Fiorentini, E. Lisi, S. Sarkar and F.L. Villante, astro-ph/9803177.

[7] R.V. Wagoner, Ap. J. Suppl. 18 (1969) 247; Ap. J. 179 (1973) 343;

L. Kawano, preprint FERMILAB-Pub-88/34-A; preprint FERMILAB-Pub-92/04-A.

[8] A. Sirlin, Phys. Rev. 164 (1967) 1767.

[9] W.J. Marciano and A. Sirlin, Phys. Rev. Lett. 56 (1986) 22, and references therein.

[10] W.J. Marciano and A. Sirlin, Phys. Rev. Lett. 46 (1981) 163.

[11] D.M. Wilkinson, Nucl. Phys. A377 (1982) 474.

[12] C. Caso et al., Eur. Phys. Jour. C3 (1998) 1.

[13] D.A. Dicus, E.W. Kolb, A.M. Gleeson, E.C.G. Sudarshan, V.L. Teplitz and M.S. Turner, Phys. Rev. D26 (1982) 2694.

[14] J.L. Cambier, J.R. Primack and M. Sher, Nucl. Phys. B209 (1982) 372.

[15] L. Dolan and R. Jackiw, Phys. Rev. D9 (1974) 3320.

[16] J.F. Donoghue, B.R. Holstein and R.W. Robinett, Ann. Phys. (N.Y.) 164 (1985) 23.

[17] J.F. Donoghue and B.R. Holstein, Phys. Rev. D28 (1983) 340; Phys. Rev. D29 (1984) 3004 .

[18] A.E. Johansson, G. Peresutti and B.S. Skagerstam, Nucl. Phys. B278 (1986) 324.

[19] W. Keil, Phys. Rev. D40 (1989)1176. 
[20] R. Baier, E. Pilon, B. Pire and D. Schiff, Nucl. Phys. B336 (1990) 157.

[21] W. Keil and R. Kobes, Physica A158 (1989) 47.

[22] M. LeBellac and D. Poizat, Z. Phys. C47 (1990) 125.

[23] T. Altherr and P.Aurenche, Phys. Rev. D40 (1989) 4171.

[24] R.L. Kobes and G.W. Semeneff, Nucl. Phys. B260 (1985) 714; ibidem B272 (1986) 329.

[25] R.F. Sawyer, Phys. Rev. D53 (1996) 4232.

[26] I.A. Chapman, Phys. Rev. D55 (1997) 6287.

[27] S. Esposito, G. Mangano, G. Miele and O. Pisanti, Phys. Rev. D58 (1998) 105023.

[28] M.A. Herrera and S. Hacyan. Ap. J. 336 (1989) 539;

N.C. Rana and B. Mitra, Phys. Rev. D44 (1991) 393;

S. Dodelson and M.S. Turner, Phys. Rev. D46 (1992) 3372;

A.D. Dolgov and M. Fukugita, Phys. Rev. D46 (1992) 5378;

N.Y. Guedin and O.Y. Guedin, astro-ph/9712199;

S. Hannestad and J. Madsen, Phys. Rev. D52 (1995) 1764;

A.D. Dolgov, S.H. Hansen and D.V. Semikoz, Nucl. Phys. B503 (1997) 426;

B. Fields, S. Dodelson and M.S. Turner, Phys. Rev. D47 (1993) 4309.

[29] D. Seckel, preprint BA-93-16, hep-ph/9305311;

R. E. Lopez, M. S. Turner and G. Gyuk, Phys. Rev. D56 (1997) 3191.

[30] V.P. Gudkov and K. Kubodera, nucl-th/9706074.

[31] F. Halzen and A.D. Martin, Quarks and Leptons, (Wiley, 1984, New York).

[32] S. Esposito, G. Mangano, G. Miele and O. Pisanti, in preparation.

[33] J.Bernstein, L.S. Brown and G. Feinberg, Rev. Mod. Phys. 61 (1989) 25.

[34] R.E. Lopez and M.S. Turner, preprint FERMILAB-Pub-98/232-A (1998), astro$\mathrm{ph} / 9807279$. 
[35] A.J. Heckler, Phys. Rev. D49 (1994) 611.

[36] K. Enqvist, K. Kainulainen and V. Semikoz, Nucl. Phys. B374 (1992) 392. 


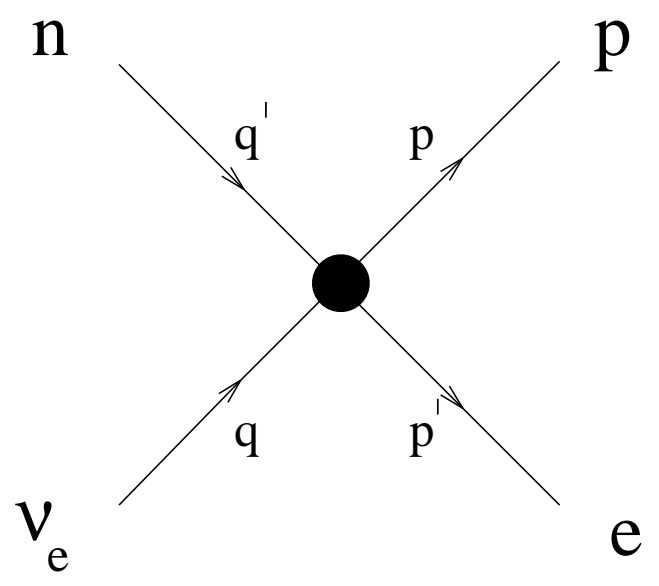

Figure 1: The Feynman diagram for the reaction $\nu_{e}+n \rightarrow e^{-}+p$ at tree level. 


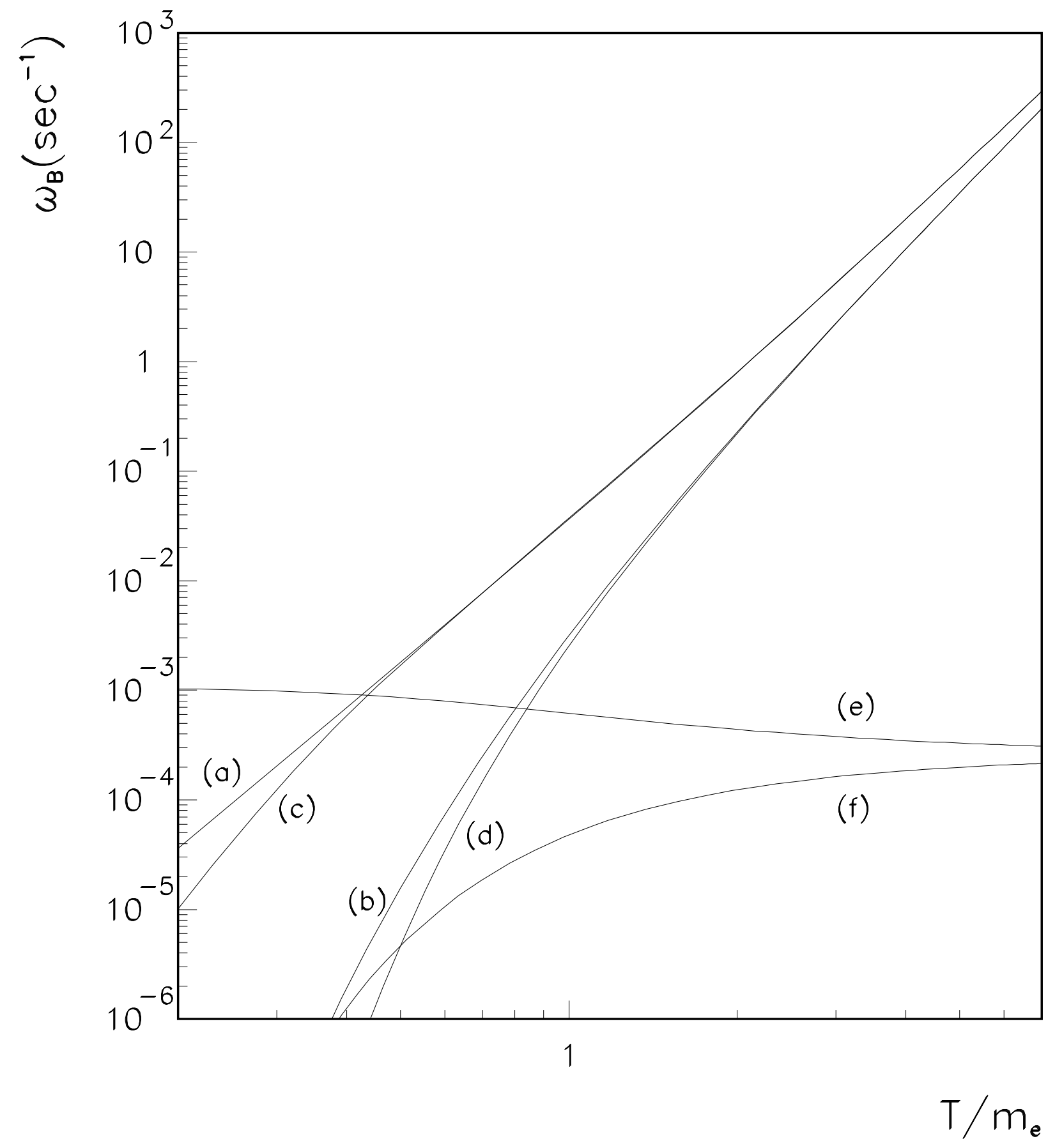

Figure 2: The Born rates $\omega_{B}$ for all $n \leftrightarrow p$ processes (see sect. 2). Hereafter the curves correspond to the reactions as labelled in (1.2) . 


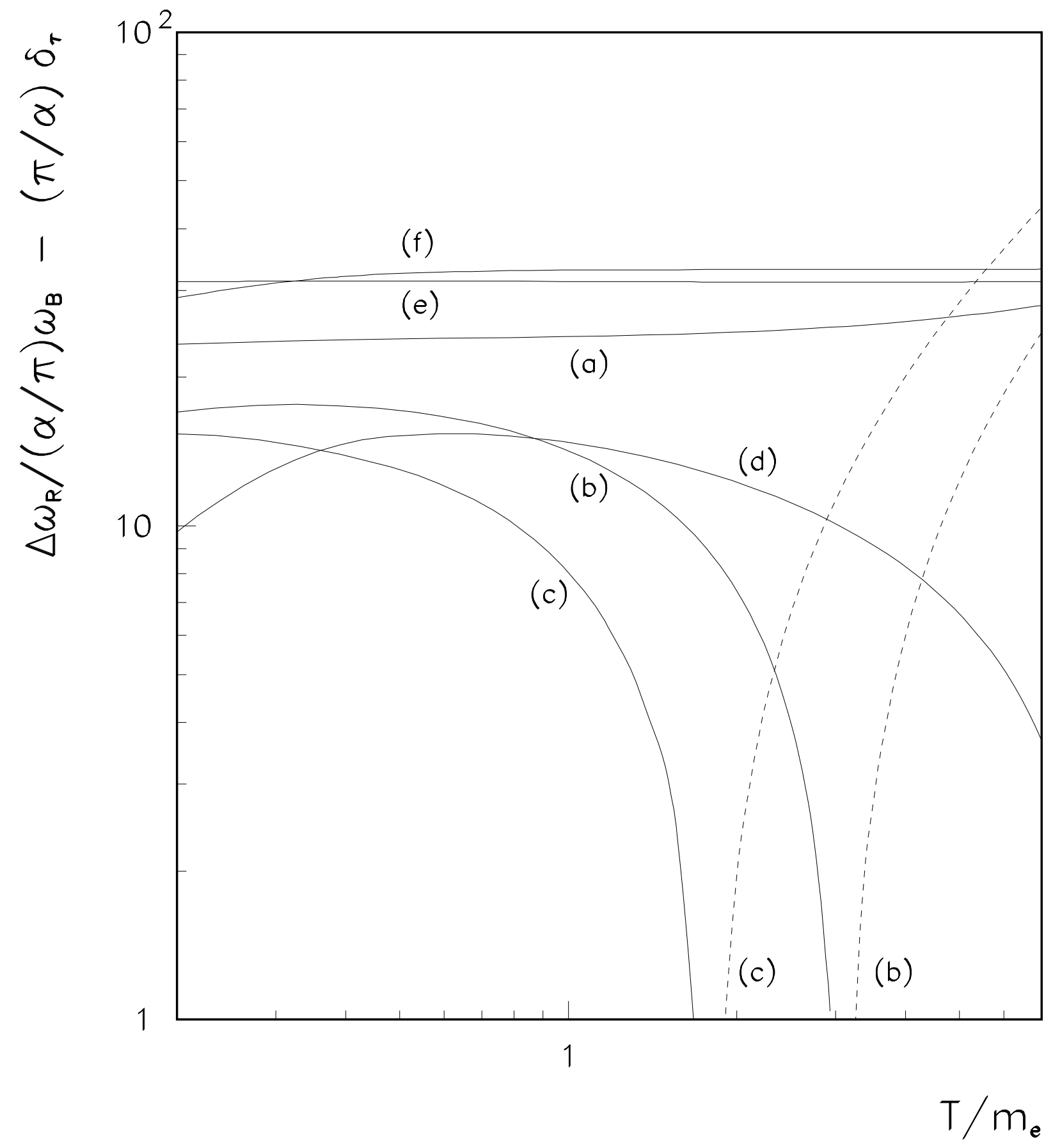

Figure 3: The quantity $\Delta \omega_{R} /(\alpha / \pi) \omega_{B}-\pi \delta_{\tau} / \alpha$ (see sect. 4.1) versus photon temperature. Hereafter dashed lines correspond to negative values. 


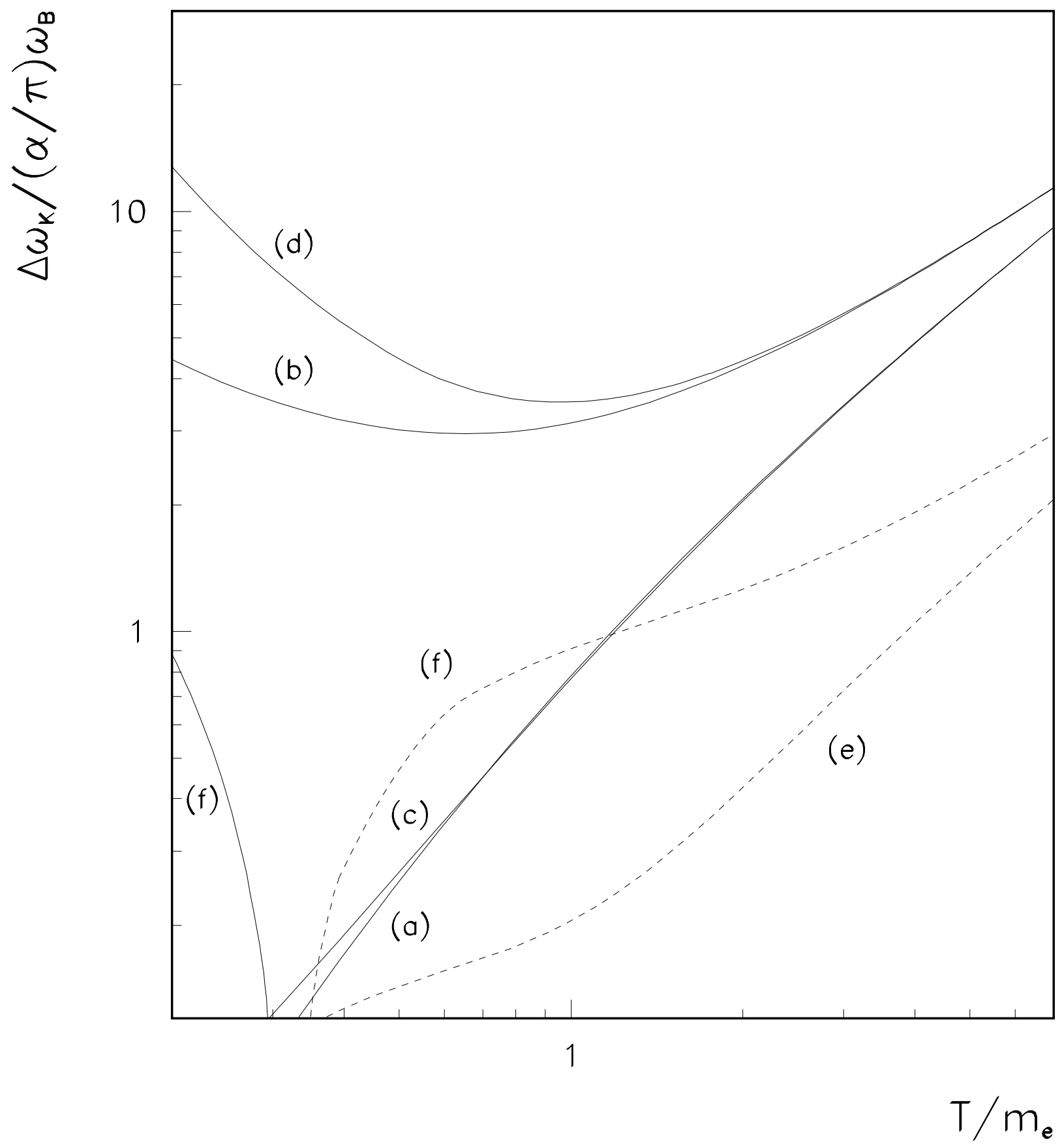

Figure 4: The ratio $\Delta \omega_{K} /(\alpha / \pi) \omega_{B}$ (see sect. 4.2). 


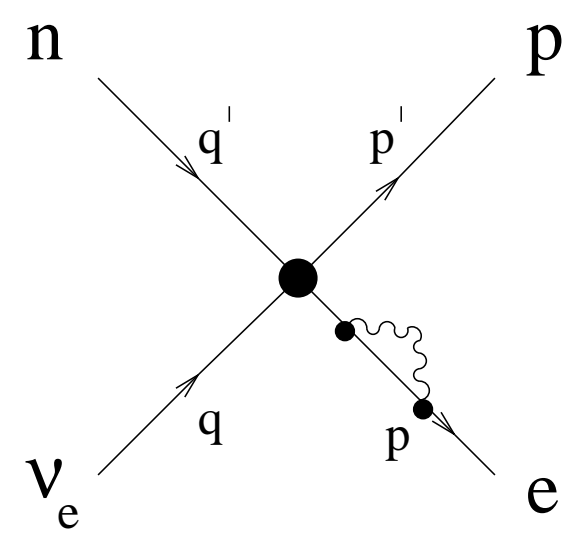

a)

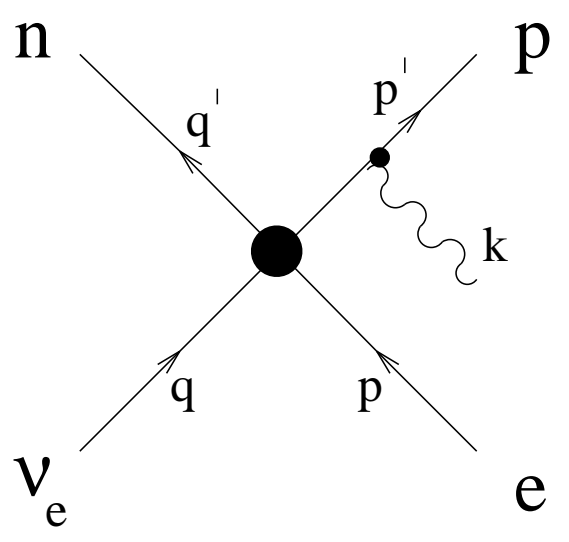

c)

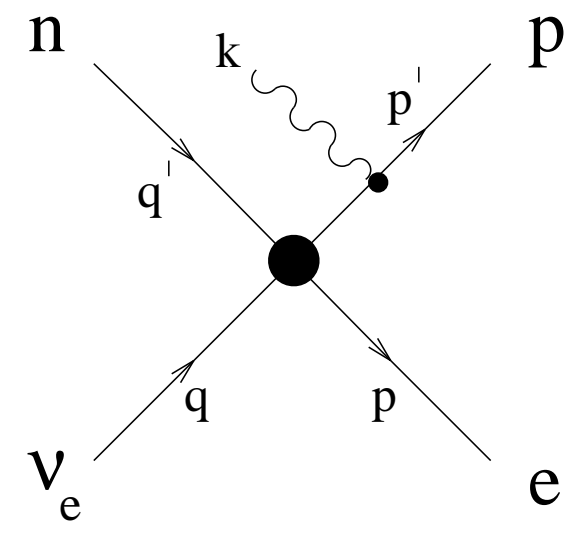

e)

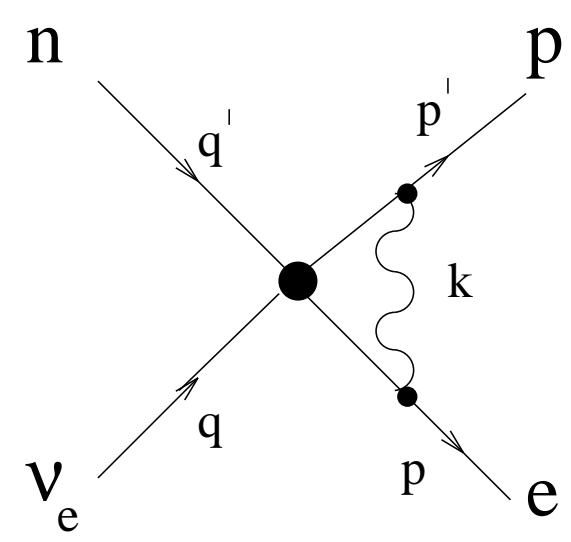

b)

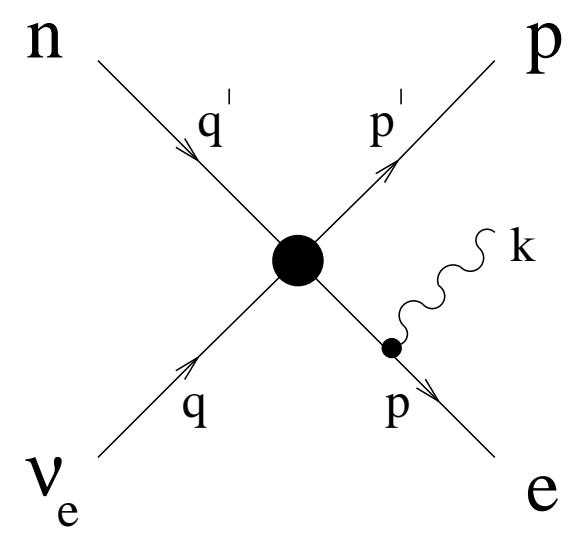

d)

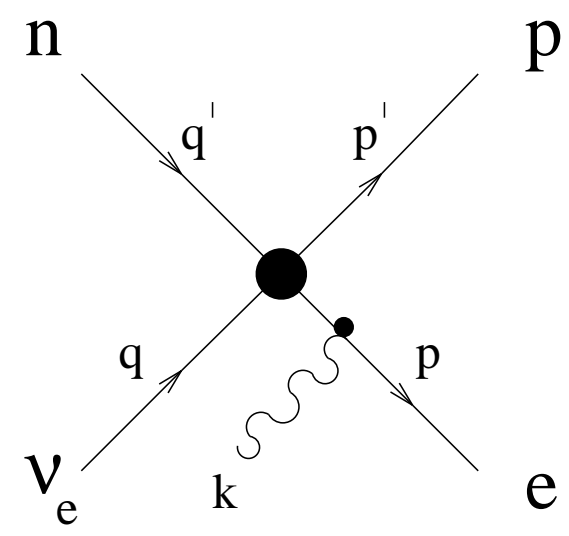

f)

Figure 5: The one-loop and photon emission/absorption diagrams for the reaction $\nu_{e}+$ $n \rightarrow e^{-}+p$. 


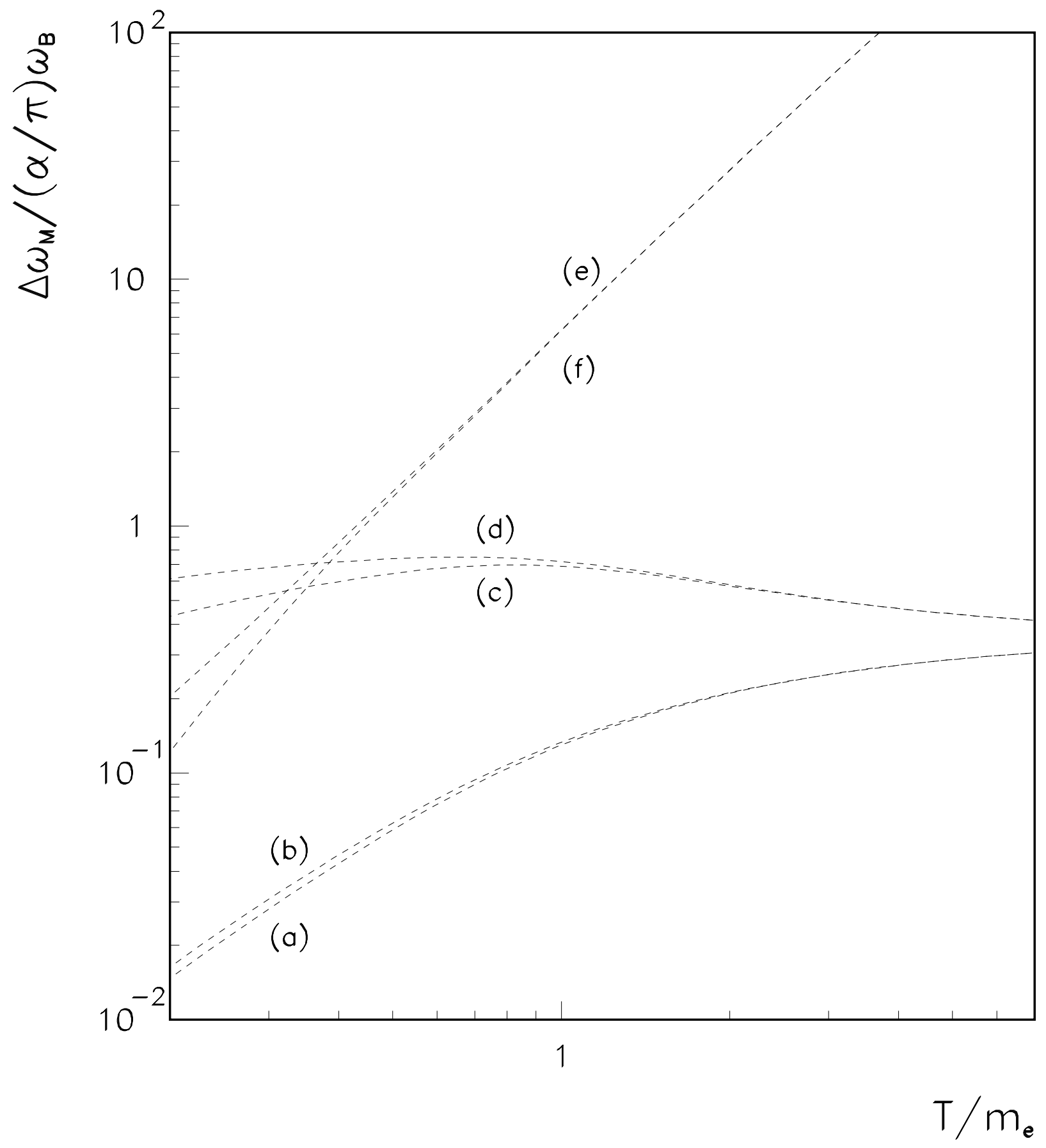

Figure 6: The ratio $\Delta \omega_{M} /(\alpha / \pi) \omega_{B}$ (see sect. 5.1). 


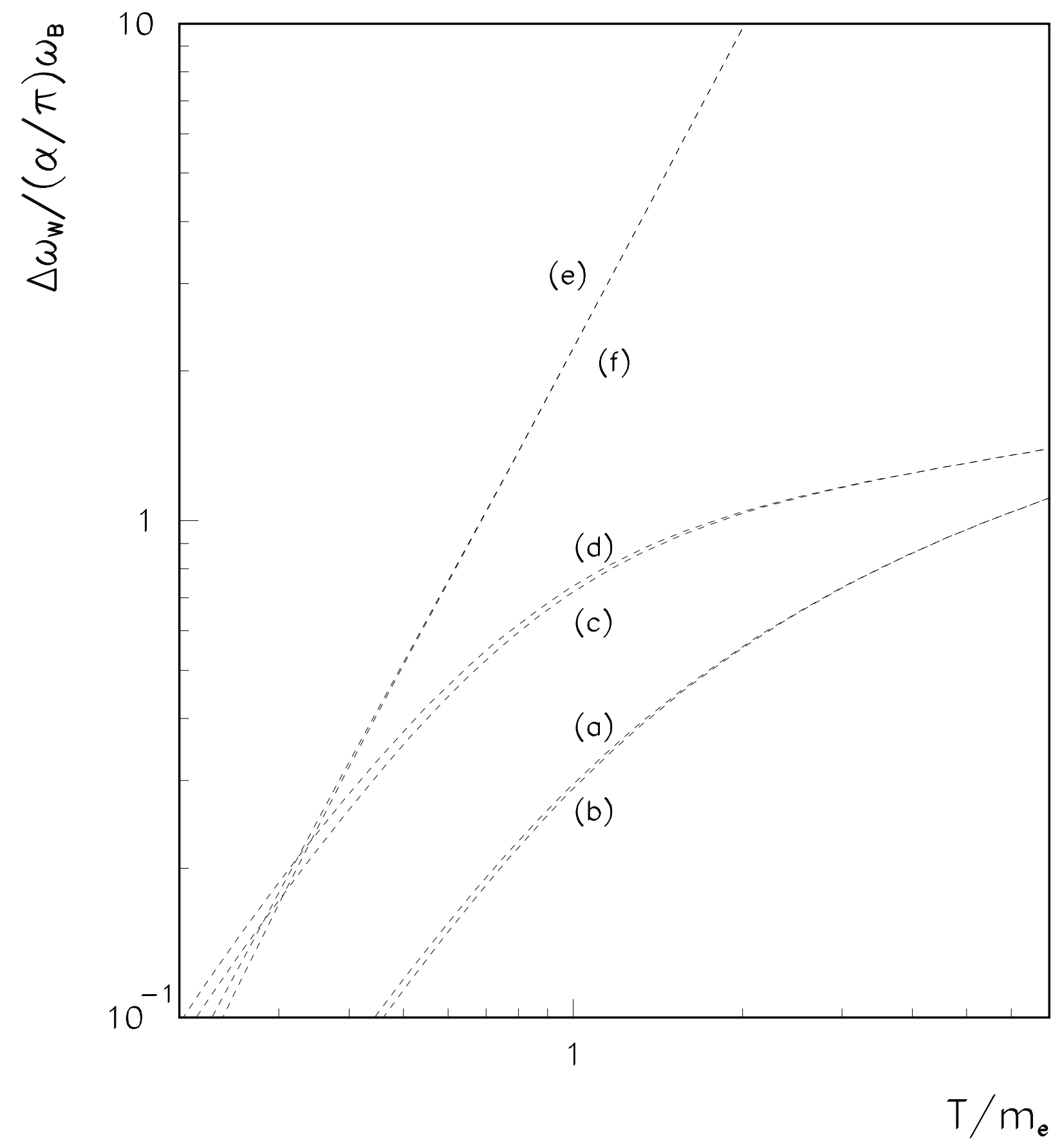

Figure 7: The ratio $\Delta \omega_{W} /(\alpha / \pi) \omega_{B}$ (see sect. 5.1). 


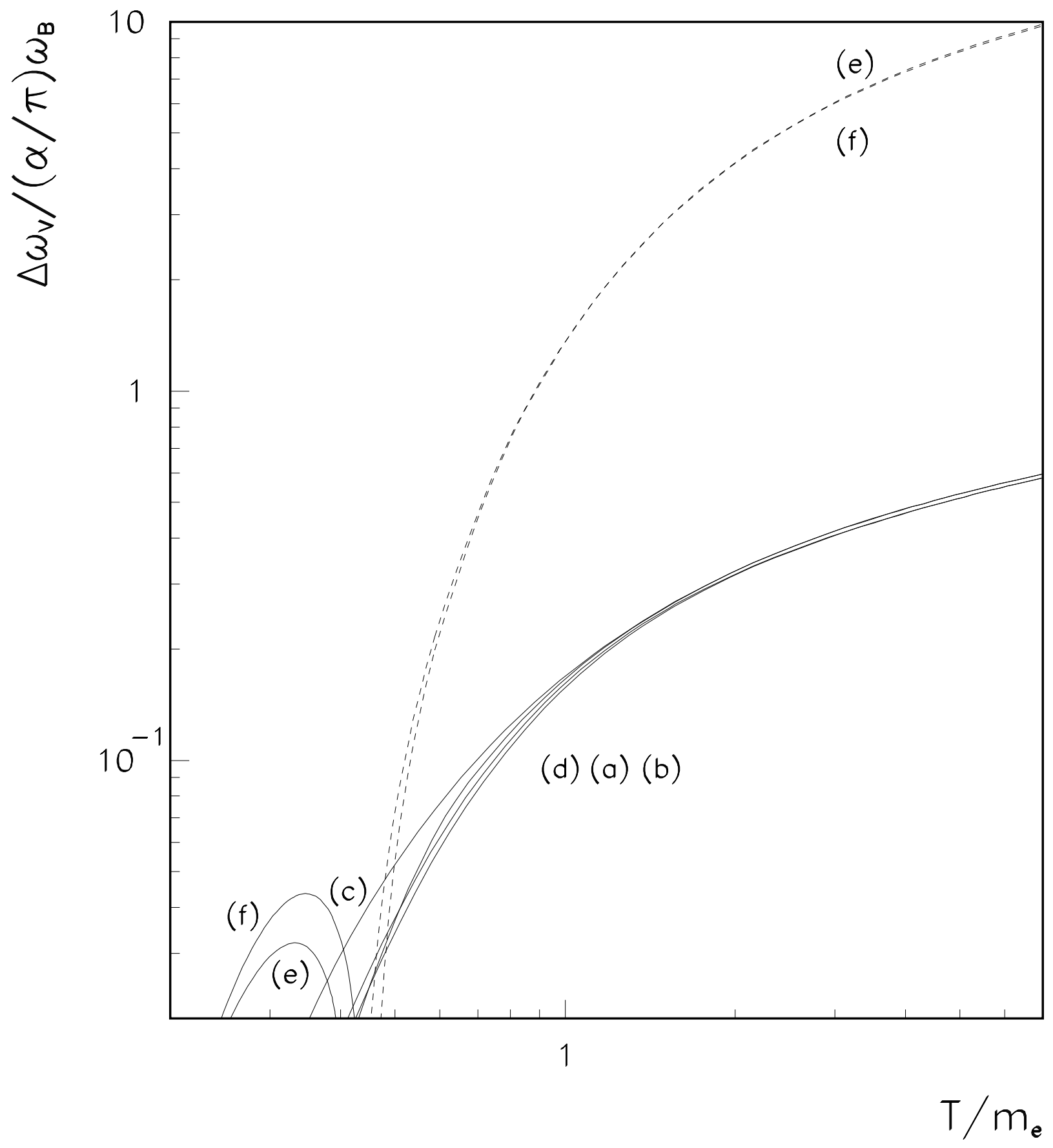

Figure 8: The ratio $\Delta \omega_{V} /(\alpha / \pi) \omega_{B}$ (see sect. 5.2). 


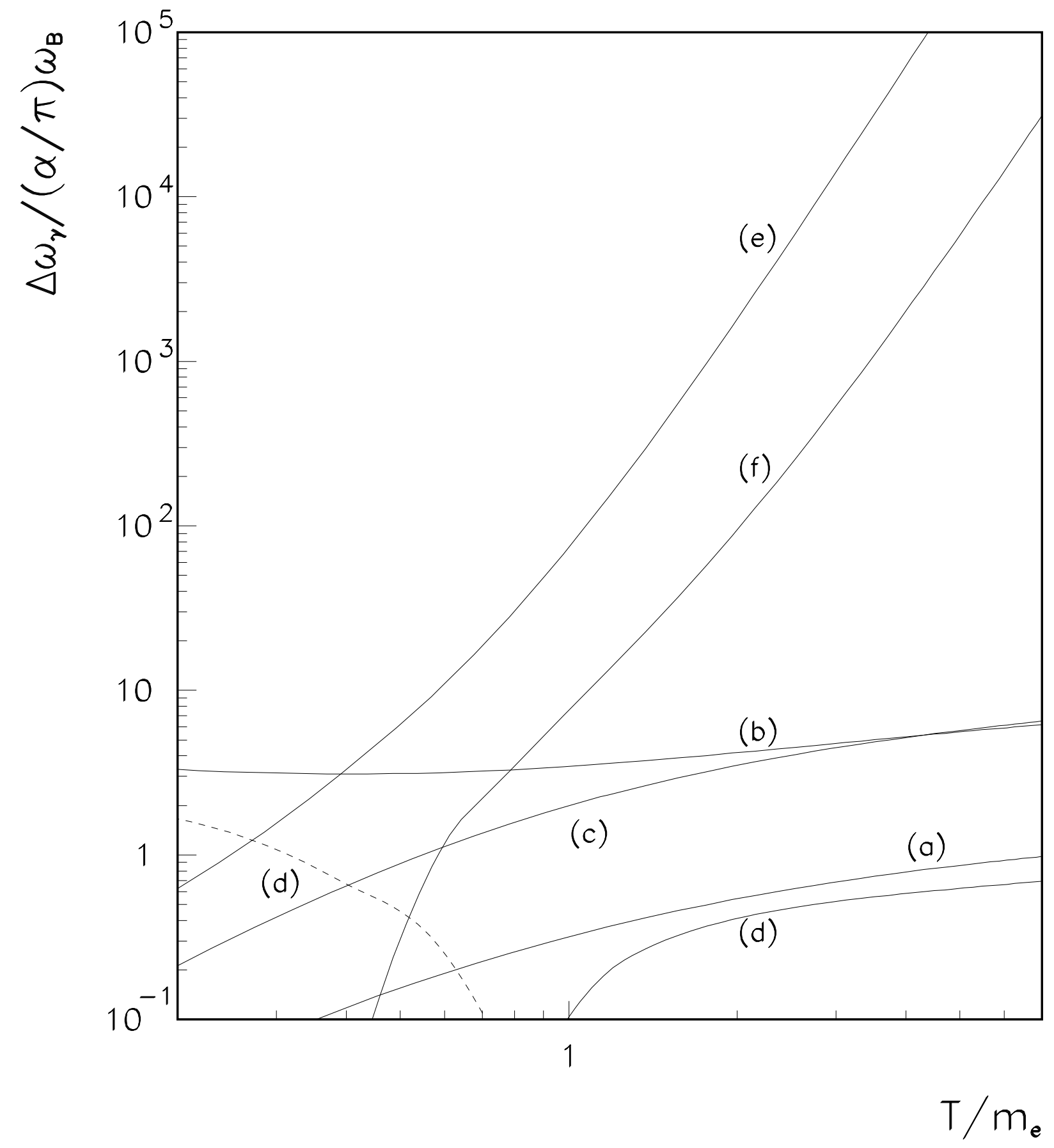

Figure 9: The ratio $\Delta \omega_{\gamma} /(\alpha / \pi) \omega_{B}$ (see sect. 5.3). 


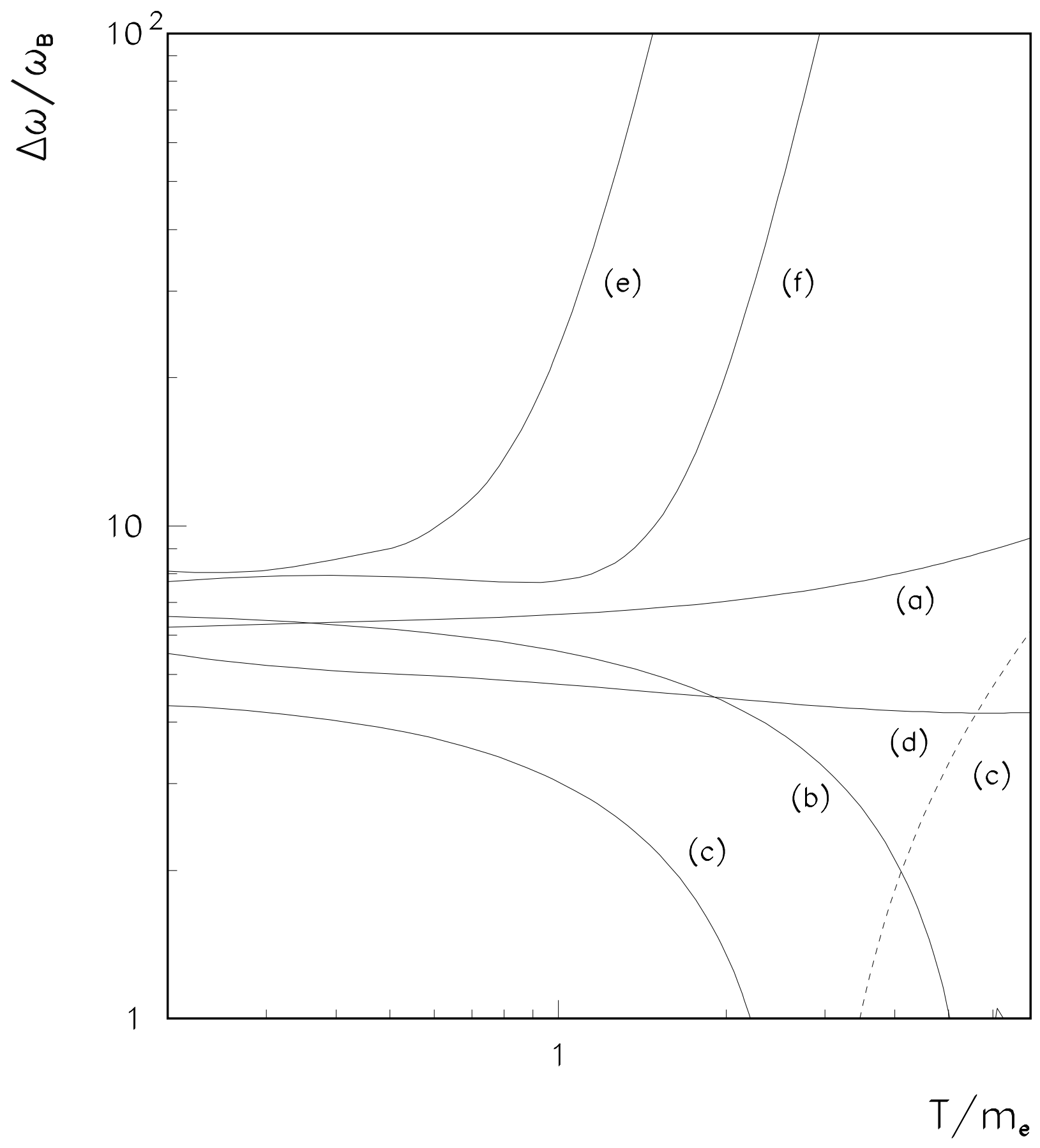

Figure 10: The total relative correction to the Born rates, in percent, are shown for the six processes $(a)-(f)$. 


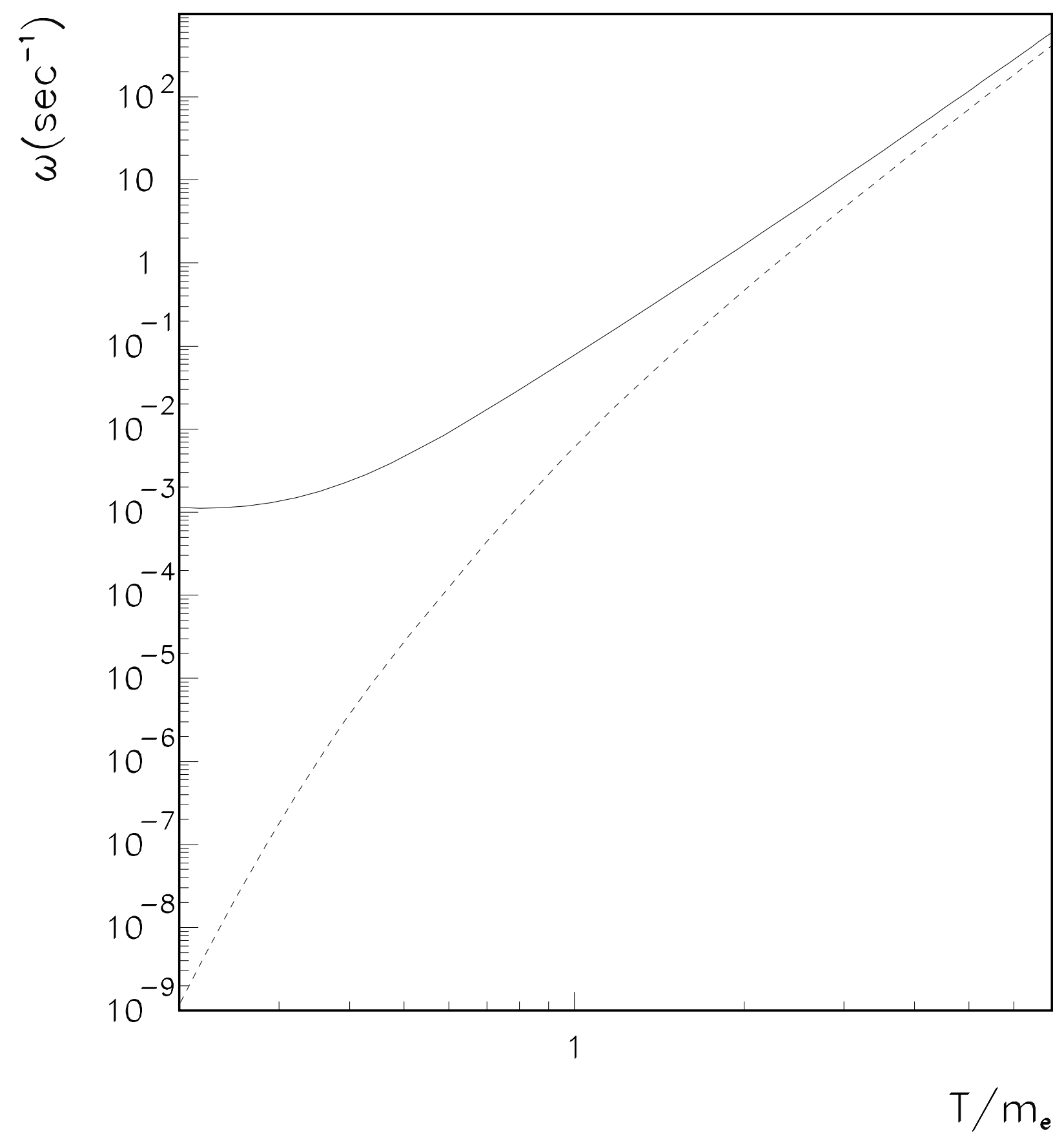

Figure 11: The total rates $\omega(n \rightarrow p)$ (solid line) and $\omega(p \rightarrow n)$ (dashed line), including all radiative, finite mass and thermal corrections. 


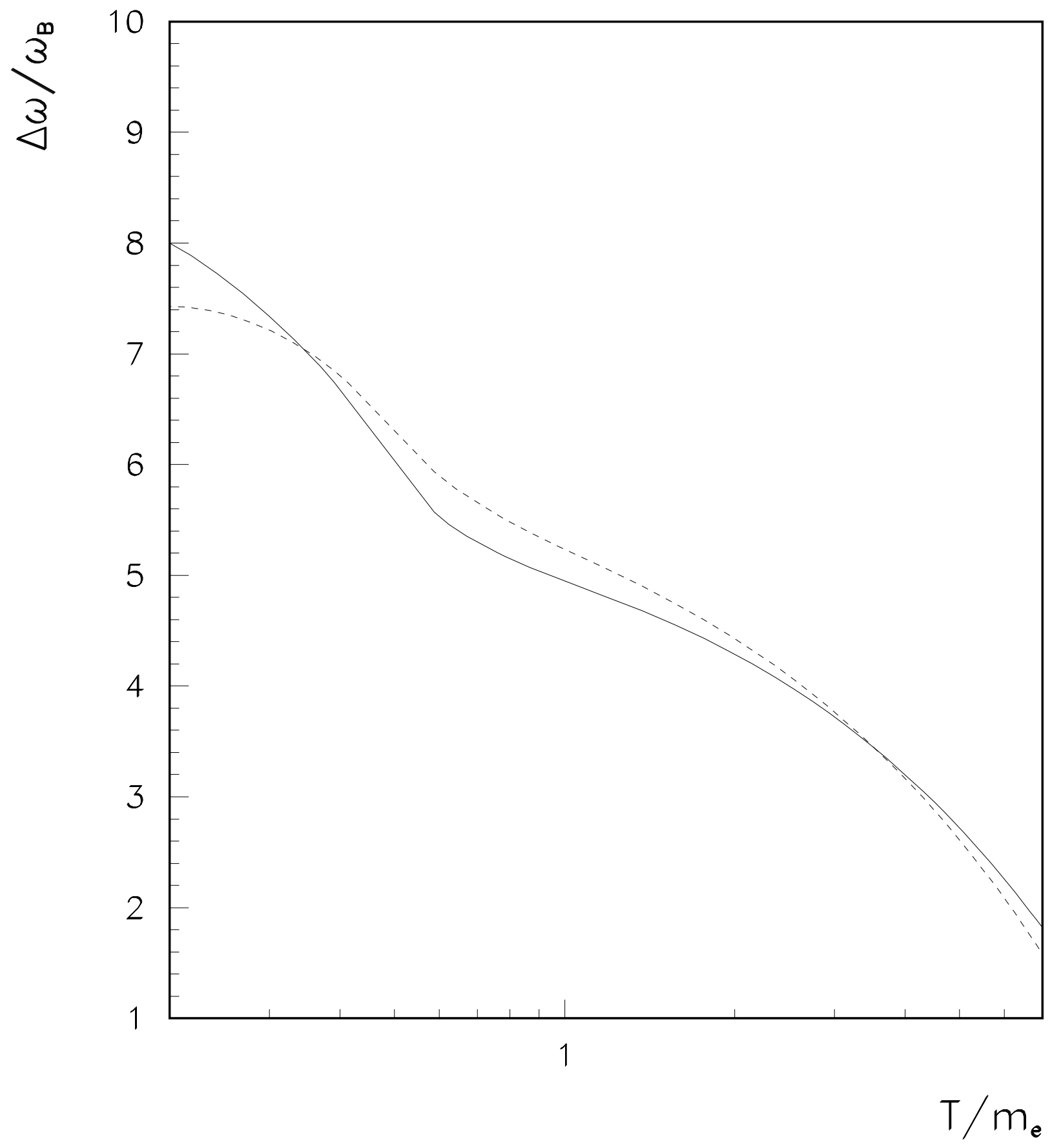

Figure 12: The total relative corrections $\Delta \omega / \omega_{B}$ for the $n \rightarrow p$ (solid line) and $p \rightarrow n$ (dashed line) processes, expressed in percent. 


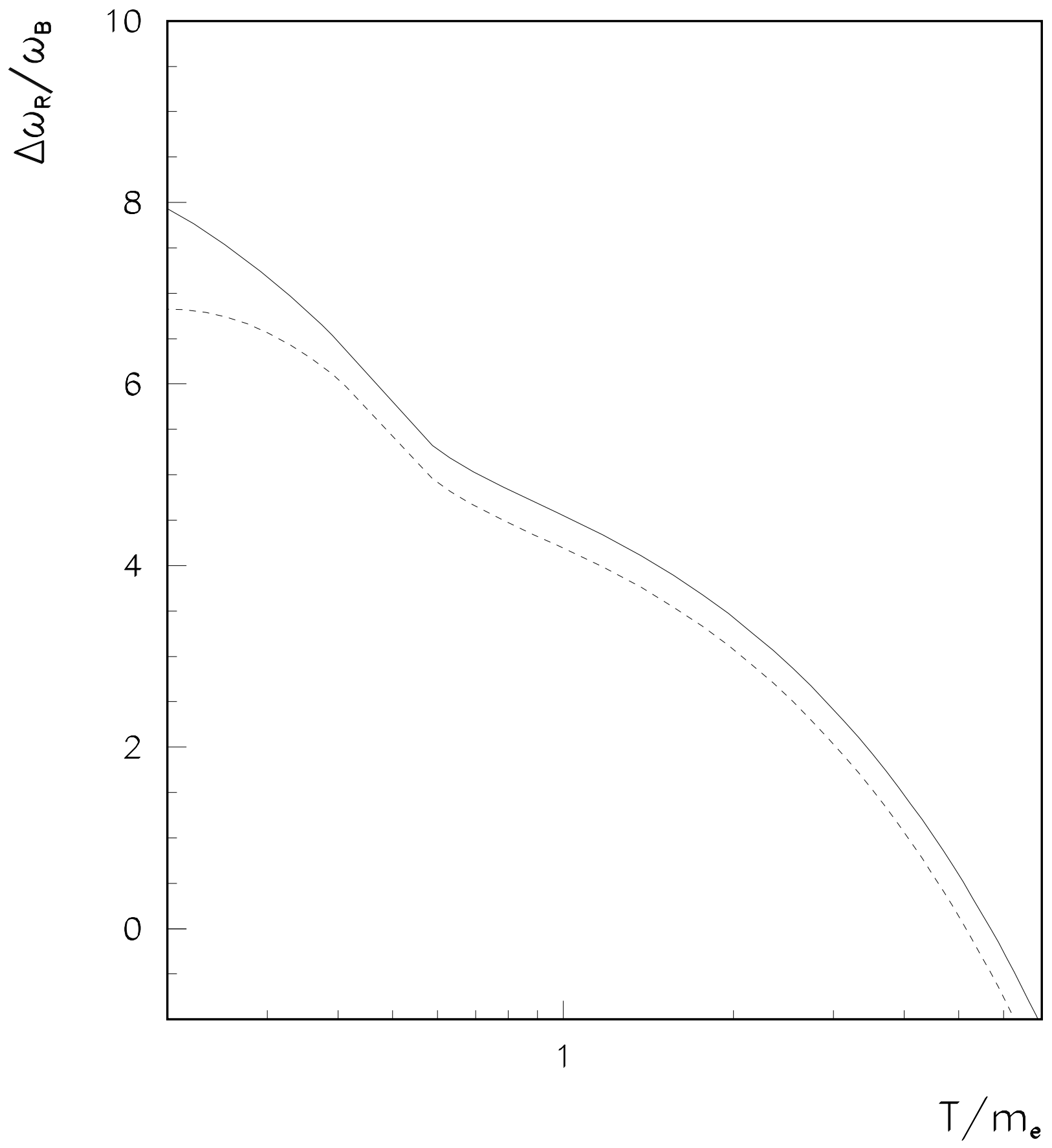

Figure 13: The zero temperature radiative corrections, for $n \rightarrow p$ (solid line) and $p \rightarrow n$ (dashed line) processes. Finite mass contributions coming from phase space integration and weak magnetism are also included. The result is expressed in percent. 


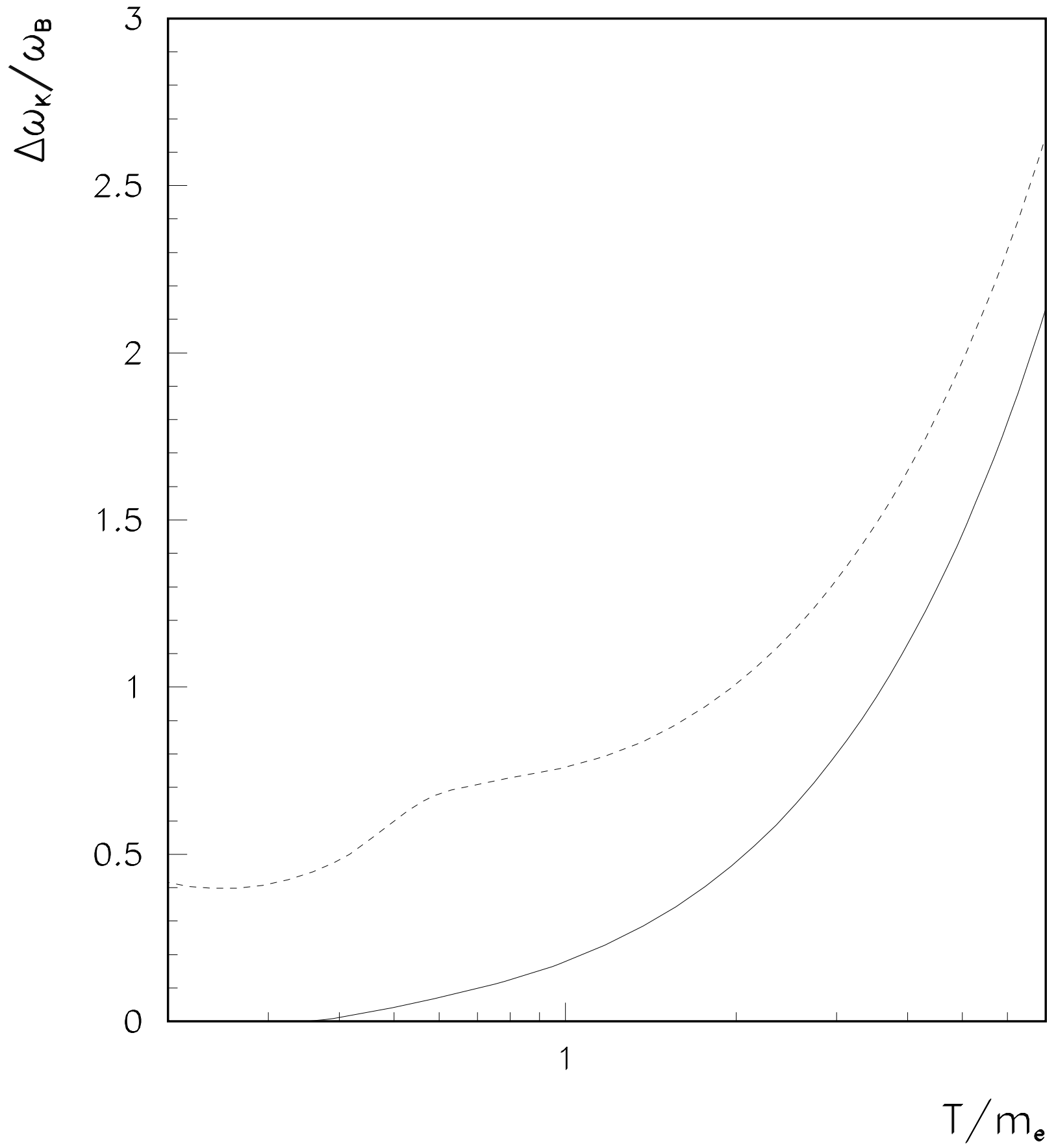

Figure 14: The kinematical contributions to the $n \rightarrow p$ (solid line) and $p \rightarrow n$ (dashed line) processes, normalized to the Born rates, in percent. 


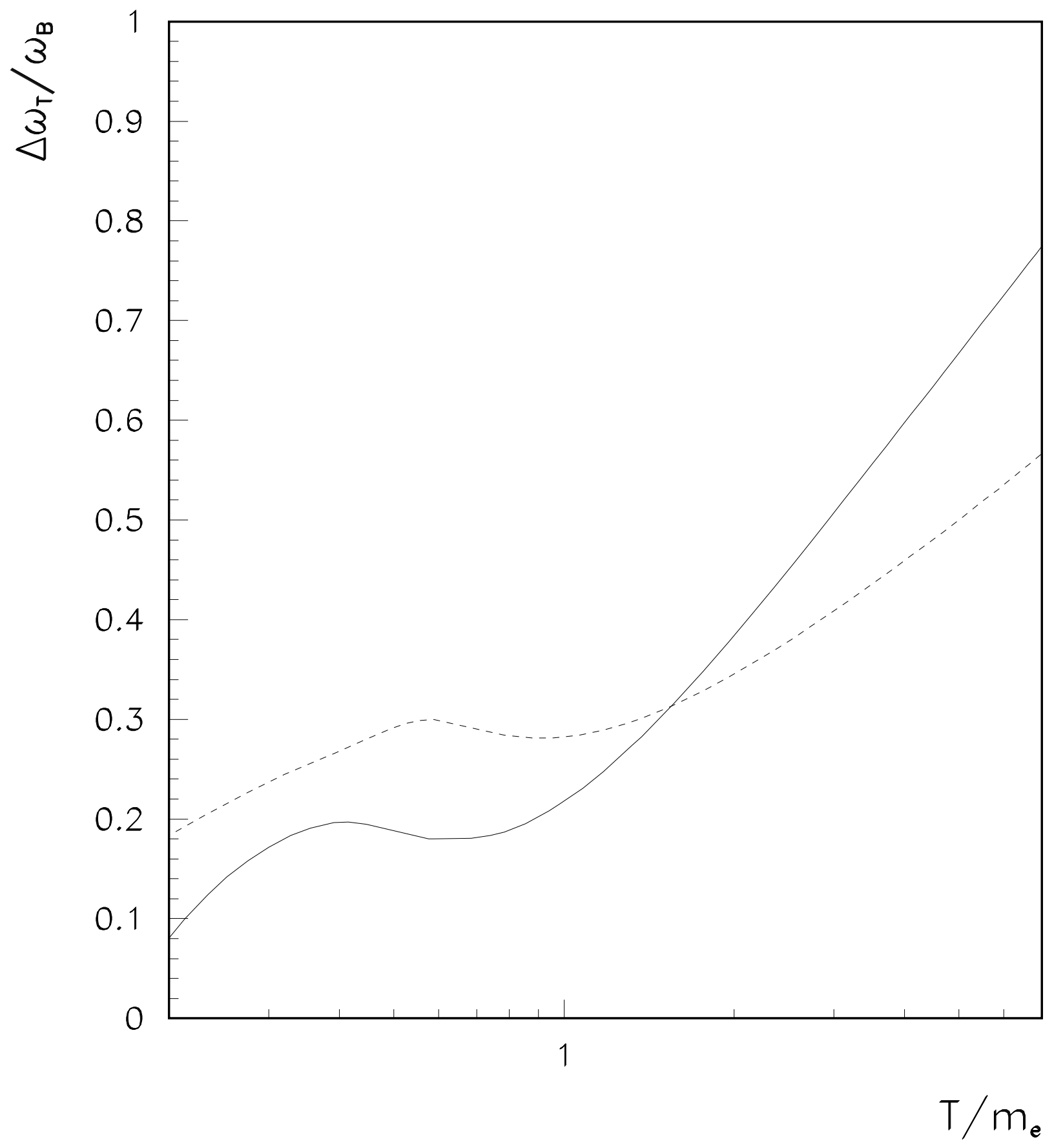

Figure 15: The whole thermal radiative corrections to the $n \rightarrow p$ (solid line) and $p \rightarrow n$ (dashed line) processes, expressed in percent. 